\title{
Carleman estimates for the time-fractional advection-diffusion equations and applications
}

\author{
Zhiyuan $\mathrm{LI}^{\dagger} \quad$ Xinchi HUANG ${ }^{\dagger}$ Masahiro YAMAMOTO ${ }^{\dagger}$
}

\begin{abstract}
In this article, we prove Carleman estimates for the generalized time-fractional advectiondiffusion equations by considering the fractional derivative as perturbation for the first order timederivative. As a direct application of the Carleman estimates, we show a conditional stability of a lateral Cauchy problem for the time-fractional advection-diffusion equation, and we also investigate the stability of an inverse source problem.
\end{abstract}

Keywords time-fractional advection-diffusion equation, Carleman estimate, lateral Cauchy problem, inverse source problem

AMS Subject Classifications 35R11, 35R30, 35B35

\section{Introduction and main results}

Recently, the position of the advection-diffusion equations (ADE) as models in a range of problems in analyzing mass transport has been challenged by more and more experiment data. For example, numerous field experiments for the solute transport in highly heterogeneous media demonstrate that solute concentration profiles exhibited anomalous non-Fickian growth rates, skewness and long-tailed profile (See e.g., 2 and [12], which are poorly characterized by the conventional mass transport equations based on Fick's law. To more accurately interpret these effects, the non-Fickian diffusion model has been proposed to mass transport model, say, time-fractional diffusion equation (FDE):

$$
\partial_{t} u(x, t)+\gamma \partial_{t}^{\alpha} u(x, t)=\Delta u(x, t), \quad(x, t) \in \mathbb{R}^{n} \times(0, \infty),
$$

where for $\alpha \in(0,1)$, by $\partial_{t}^{\alpha}$ we denote the Caputo derivative with respect to temporal variable $t>0$ :

$$
\partial_{t}^{\alpha} g(t)=\frac{1}{\Gamma(1-\alpha)} \int_{0}^{t}(t-\tau)^{-\alpha} \frac{\mathrm{d} g(\tau)}{\mathrm{d} \tau} \mathrm{d} \tau
$$

where $\Gamma(\cdot)$ is the usual Gamma function. See, e.g., 9] and [14 for the properties of the Caputo derivative, and see, e.g., [5], [16] and the references therein for the FDEs.

Introducing the time-fractional derivatives of arbitrary order into the equation of mass transport for a heterogeneous medium achieved great successes, for example, it is shown to be an efficient model for describing some anomalous diffusion processes in the highly heterogeneous media by [6] in which the authors pointed out that diffusion equation with time-fractional derivative was well-performed in describing the long-tailed profile of a particle diffuses in a highly heterogeneous medium, and by [13. where the theoretical fractional calculus on FDEs shows that there holds the non-Fick's law in the anomalous diffusion. We also refer to [16] in which the MADE site mobile tritium mass decline is well modeled by the equation (1) with the time-fractional derivative of order $\alpha=0.33$.

In this paper, assuming $0<\alpha_{\ell}<\cdots<\alpha_{1}<1$, we consider a generalized time-fractional advectiondiffusion equation (FADE)

$$
(L u)(x, t) \equiv \partial_{t} u+\sum_{j=1}^{\ell} q_{j}(x, t) \partial_{t}^{\alpha_{j}} u-\sum_{i, j=1}^{n} a_{i j}(x, t) \partial_{i} \partial_{j} u+\sum_{i=1}^{n} b_{i}(x, t) \partial_{i} u+c(x, t) u=F,
$$

\footnotetext{
Manuscript last updated: October 10, 2018.

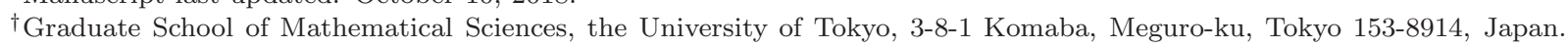

E-mail: zyli@ms.u-tokyo.ac.jp, huangxc@ms.u-tokyo.ac.jp, myama@ms.u-tokyo.ac.jp.
} 
where $(x, t)$ in $\mathbb{R}^{n} \times(0, \infty)$. The above equation (2) has the spatially and temporally variable coefficients, and such kind of equations simulate the advection diffusion, which is more general than that in [5] and [16], and so can be regarded as more feasible model equation than symmetric fractional diffusion equations in modeling diffusion in heterogeneous media. Here in this article, we study the stability for a lateral Cauchy problem and the stability for an inverse source problem for this equation, and the stability is a fundamental theoretical subject. To the best knowledge of the authors, except the special case that $\alpha=1 / 2$ which is discussed in [8], the stability results for both of the lateral Cauchy problem and inverse source problem of the equation (2) were not yet established. Here it should be mentioned that in the general order case, the transform argument and Fourier methods used in the above mentioned references [5] and [16] can not work any more because of the non-symmetry of the system and $t$-dependent coefficients, and it is very complicated to follow the treatment used in 8 even for $\alpha=1 / 3$. One of the reasons is that in this case one needs to establish a Carleman estimate for parabolic operators of order 6 in the space variables, which will cause a huge amount of computation. However, from the shape of the equation (22) we regard the lower fractional order term as a perturbation of the first order time-derivative, which enable one to derive the Carleman estimate for the equation (2) in the framework of the Carleman estimate for the parabolic equations, and then consider the stability of the lateral Cauchy problem and inverse source problem.

To this end, we start from fixing some general settings and notations. Let $T>0$ be fixed constant and $\Omega \subset \mathbb{R}^{n}$ is a bounded domain, $n \geq 1$, with sufficiently smooth boundary $\partial \Omega$, for example, of $C^{2}$-class. We set $Q:=\Omega \times(0, T)$. Assume that $a_{i j}=a_{j i} \in C^{1}(\bar{Q}), 1 \leq i, j \leq n$, satisfies that

$$
\rho \sum_{j=1}^{n} \xi_{j}^{2} \leq \sum_{j, k=1}^{n} a_{j k}(x, t) \xi_{j} \xi_{k}, \quad(x, t) \in \bar{Q}, \quad \xi \in \mathbb{R}^{n},
$$

where $\rho>0$ is a constant independent of $x, t, \xi$. We set $\partial_{\nu_{A}} u=\sum_{i, j=1}^{n} a_{i j} \nu_{i} \partial_{j} u$ where $\left(\nu_{1}, \cdots, \nu_{n}\right)$ denotes the unit outwards normal vector to the boundary $\partial \Omega$. Let $L^{2}(\Omega)$ and $H^{k, \ell}(Q)(k \geq 0, \ell \geq 0)$ denote Sobolev spaces (See, e.g., [1] and [18). Similar to Theorem 5.1 in [18, for arbitrary non-empty relatively open sub-boundary $\Gamma \subset \partial \Omega$, we choose a bounded domain $\Omega_{1}$ with sufficiently smooth boundary such that

$$
\Omega \subsetneq \Omega_{1}, \quad \bar{\Gamma}=\overline{\partial \Omega \cap \Omega_{1}}, \quad \partial \Omega \backslash \Gamma \subset \partial \Omega_{1} .
$$

We then apply Lemma 4.1 in 18 to find a function $d \in C^{2}\left(\overline{\Omega_{1}}\right)$ satisfying

$$
d>0 \text { in } \Omega, \quad|\nabla d|>0 \text { on } \bar{\Omega}, \quad d=0 \text { on } \partial \Omega \backslash \Gamma .
$$

Now let us first consider the equation (2) with $\alpha_{1}<\frac{1}{2}$ (we call the corresponding diffusion as subdiffusion), we set the weight function $\varphi_{1}$ as follows

$$
\varphi_{1}(x, t)=\mathrm{e}^{\lambda \psi_{1}(x, t)}, \quad \psi_{1}(x, t)=d(x)-\beta t^{2-2 \alpha_{1}}, \quad \forall \lambda \geq 0, x \in \overline{\Omega_{1}}, t \geq 0,
$$

where $\beta>0$ is a positive constant which will be chosen later, and we have the following Carleman type estimate for the equation (2)

Theorem 1. We assume $\alpha_{1}<\frac{1}{2}$ and $q_{i}, b_{j}, c \in L^{\infty}(Q)(i=1, \cdots, \ell, j=1, \cdots, n)$ in the equation (2), and we let $\Sigma_{0}=\bar{\Omega} \times\{0\}$ and $D \subset Q$ be bounded domain whose boundary $\partial D$ is composed of a finite number of smooth surfaces. Then there exists a constant $\lambda_{0}>0$ such that for arbitrary $\lambda \geq \lambda_{0}$, we can choose $a$ constant $s_{0}(\lambda)>0$ satisfying: there exist constants $C=C\left(s_{0}, \lambda_{0}\right)>0$ and $C(\lambda)>0$ such that

$$
\begin{aligned}
& \int_{D}\left\{\frac{1}{s \varphi_{1}}\left|\partial_{t} u\right|^{2}+s \lambda^{2} \varphi_{1}|\nabla u|^{2}+s^{3} \lambda^{4} \varphi_{1}^{3} u^{2}\right\} \mathrm{e}^{2 s \varphi_{1}} \mathrm{~d} x \mathrm{~d} t \\
\leq & C \int_{D}|\widetilde{L} u|^{2} \mathrm{e}^{2 s \varphi_{1}} \mathrm{~d} x \mathrm{~d} t+C(\lambda) \mathrm{e}^{C(\lambda) s} \int_{\partial D}\left(|\nabla u|^{2}+u^{2}\right) \mathrm{d} \Sigma+C(\lambda) \mathrm{e}^{C(\lambda) s} \int_{\partial D \backslash \Sigma_{0}}\left|\partial_{t} u\right|^{2} \mathrm{~d} \Sigma
\end{aligned}
$$

for all $s \geq s_{0}$ and all $u \in H^{2,1}(D)$, where $\widetilde{L}:=L-\sum_{j=1}^{\ell} q_{j}(x, t) \partial_{t}^{\alpha_{j}}$.

From the above Carleman estimate, similar to the argument used in [18, we further have the conditional stability for the lateral Cauchy problem for the equation (21). However, a bit different from the results in [18, here due to the choice of the weight function in the derivation of the Carleman estimate in Theorem 1 . we can only prove that the continuous dependency of the solution with respect to initial values, boundary values and source terms in the case of $\alpha_{1} \in\left(0, \frac{1}{2}\right)$, say, 
Theorem 2. We assume $\alpha_{1}<\frac{1}{2}$ and $q_{i}, b_{j}, c \in L^{\infty}(Q)(i=1, \cdots, \ell, j=1, \cdots, n)$ in the equation (2). Let $\Gamma \subset \partial \Omega$ be an arbitrary non-empty relatively open sub-boundary of $\partial \Omega$. For any bounded domain $\Omega_{0}$ such that $\overline{\Omega_{0}} \subset \Omega \cup \Gamma, \partial \Omega_{0} \cap \partial \Omega \subsetneq \Gamma$ is a non-empty open subset of $\partial \Omega$, we can choose a sufficiently small $\varepsilon=\varepsilon\left(T, \Omega_{0}\right)>0$ such that

$$
\|u\|_{H^{1,1}\left(\Omega_{0} \times(0, \varepsilon)\right)} \leq C\|u\|_{H^{1,1}(Q)}^{1-\theta} \mathcal{D}^{\theta}
$$

where $\mathcal{D}:=\|u(\cdot, 0)\|_{H^{1}(\Omega)}+\|F\|_{L^{2}(Q)}+\|u\|_{H^{1}(\Gamma \times(0, T))}+\left\|\partial_{\nu_{A}} u\right\|_{L^{2}(\Gamma \times(0, T))}$, and the constants $C>0$ and $\theta \in(0,1)$ may depend on $T$, the choice of $\Omega_{0}$ and the coefficients of the equation (2).

The above arguments used for deriving Theorem 2 cannot work anymore for the general fractional order $\alpha_{1} \geq \frac{1}{2}$ (the corresponding diffusion is called as sup-diffusion), but for some fractional orders, it is expected that the method of Carleman estimate still works. As a partially affirmative answer, we focus on deriving the Carleman estimate for the case of rational fractional order $\alpha_{1}$ which is smaller than $\frac{3}{4}$, i.e. $\alpha_{1} \leq \frac{3}{4}$ in the form of $\frac{m}{k}, m, k \in \mathbb{N}$. For this, we consider the following equation

$$
(L u)(x, t) \equiv \partial_{t} u+q(x) \partial_{t}^{\alpha} u-\sum_{i, j=1}^{n} a_{i j}(x) \partial_{i} \partial_{j} u+\sum_{i=1}^{n} b_{i}(x) \partial_{i} u+c(x) u=F
$$

in the case of $\alpha \in\left(0, \frac{3}{4}\right]$, where the coefficients satisfy: $b_{i} \in L^{\infty}(\Omega), i=1, \cdots, n, c \in L^{\infty}(\Omega)$ and the source term $F$ is assumed to be smooth enough. To this end, we first introduce the Riemann-Liouville fractional derivative of order $\alpha \in[m-1, m)$ with $m=1,2, \cdots$ which is usually defined by

$$
D_{t}^{\alpha} h(t):=\frac{1}{\Gamma(m-\alpha)} \frac{\mathrm{d}^{m}}{\mathrm{~d} t^{m}} \int_{0}^{t}(t-r)^{-\alpha+m-1} h(r) \mathrm{d} r
$$

and the Riemann-Liouville fractional integral $D_{t}^{-\alpha}$ of order $\alpha \in(0,1)$ which is defined by

$$
D_{t}^{-\alpha} h(t):=\frac{1}{\Gamma(\alpha)} \int_{0}^{t}(t-r)^{\alpha-1} h(r) \mathrm{d} r .
$$

We choose the weight function $\varphi_{2}$ :

$$
\varphi_{2}(x, t)=e^{\lambda \psi_{2}(x, t)}, \quad \psi_{2}(x, t)=d(x)-\beta\left(t-t_{0}\right)^{2}+c_{0},
$$

where $t_{0} \in(0, T)$ and $\beta>0$ are to be fixed later and $c_{0}:=\max \left\{\beta t_{0}^{2}, \beta\left(T-t_{0}\right)^{2}\right\}$ guarantees the nonnegativeness of the function $\psi_{2}$. In needs of applications, we establish a special Carleman estimate with a cut-off function $\chi_{0} \in C^{\infty}\left(\mathbb{R}^{n+1}\right)$ which is defined by

$$
\chi_{0}(x, t)= \begin{cases}1, & (x, t) \in D_{0} \\ 0, & \text { outside of } D\end{cases}
$$

where $D$ is an arbitrary subdomain of $Q_{1}:=\Omega_{1} \times(0, T)$ and $D_{0} \subsetneq D$. Now we are ready to state the Carleman estimate.

Theorem 3. For any rational number $\alpha=\frac{m}{k} \leq \frac{3}{4}, m, k \in \mathbb{N}$, we assume that $D_{t}^{\frac{j}{k}} F \in L^{2}(Q)$ for $j=j_{1}, \ldots, j_{k}$ where $j_{l}:=-\frac{k}{2}+\frac{(-1)^{k}-1}{4}+l, l=1, \ldots, k$. Then there exist constants $\hat{s} \geq 1$ and $C>0$ such that

$$
\begin{aligned}
& \int_{Q} \chi_{0}^{2}\left(\sum_{i, l=1}^{n}\left(s \varphi_{2}\right)^{\frac{4}{k} j_{1}-1}\left|\partial_{i} \partial_{l} u\right|^{2}+\left(s \varphi_{2}\right)^{\frac{4}{k} j_{1}+1}|\nabla u|^{2}+\sum_{j=j_{1}}^{j_{k}+k}\left(s \varphi_{2}\right)^{-\frac{4}{k}\left(j-j_{1}\right)+3}\left|D_{t}^{\frac{j}{k}} u\right|^{2}\right) \mathrm{e}^{2 s \varphi_{2}} \mathrm{~d} x \mathrm{~d} t \\
& \leq C \int_{Q} \chi_{0}^{2}\left(\sum_{j=j_{1}}^{j_{k}}\left(s \varphi_{2}\right)^{-\frac{4}{k}\left(j-j_{1}\right)}\left|D_{t}^{\frac{j}{k}} F\right|^{2}\right) \mathrm{e}^{2 s \varphi_{2}} \mathrm{~d} x \mathrm{~d} t+L o w+B d y
\end{aligned}
$$


for all $s \geq \hat{s}$, large fixed $\lambda \geq 1$ and all $u$ smooth enough satisfying (7) and $u(x, 0)=0$ for $\forall x \in \Omega$, where Low and Bdy are defined as follows

$$
\begin{aligned}
& \text { Low }:=C s \int_{Q}\left(\left|\partial_{t} \chi_{0}\right|^{2}+\left|\nabla \chi_{0}\right|^{2}+\sum_{i, j=1}^{n}\left|\partial_{i} \partial_{j} \chi_{0}\right|^{2}\right)\left(\sum_{j=j_{1}}^{j_{k}}\left(\left|\nabla\left(D_{t}^{\frac{j}{k}} u\right)\right|^{2}+\left|D_{t}^{\frac{j}{k}} u\right|^{2}\right)\right) \mathrm{e}^{2 s \varphi_{2}} \mathrm{~d} x \mathrm{~d} t, \\
& B d y:=C \mathrm{e}^{C s} \int_{\partial Q}\left(\left|\partial_{t} \chi_{0}\right|^{2}+\left|\nabla \chi_{0}\right|^{2}+\left|\chi_{0}\right|^{2}\right)\left(\sum_{j=j_{1}}^{j_{k}}\left(\left|\nabla_{x, t}\left(D_{t}^{\frac{j}{k}} u\right)\right|^{2}+\left|D_{t}^{\frac{j}{k}} u\right|^{2}\right)\right) \mathrm{d} S \mathrm{~d} t .
\end{aligned}
$$

We notice that the above Carleman estimate with the regular weight function $\varphi_{2}$ requires the homogeneous initial condition, which possibly roots in the memory effect of time-fractional derivatives. We refer to the publications [3] and [17 for the Carleman estimate for the diffusion equations with a half order time-fractional derivative where the homogeneous initial value is also necessary for deriving the Carleman estimates. Moreover, it should be mentioned here that our method can work for multi-term case where the fractional order $\alpha_{j}, j=1, \ldots, l$ are all rational numbers and the largest one $\alpha_{1}$ is smaller than $\frac{3}{4}$.

As an application of Theorem 3, we can easily derive the stability for the lateral Cauchy problem for the equation (77). For simplicity, we only state the theorem in the critical case: $\alpha=\frac{3}{4}$. The results for the case of rational order less than $\frac{3}{4}$ can be established by using the similar argument.

Theorem 4. For any small $\epsilon>0$ and any bounded domain $\Omega_{0}$ satisfying $\overline{\Omega_{0}} \subset \Omega \cup \Gamma, \partial \Omega_{0} \cap \partial \Omega$ be a non-empty open subset of $\partial \Omega$ and $\partial \Omega_{0} \cap \partial \Omega \subsetneq \Gamma$, there exist constants $C>0$ and $\theta \in(0,1)$ such that

$$
\|u\|_{H^{2,1}\left(\Omega_{0} \times(\epsilon, T-\epsilon)\right)} \leq C \mathcal{D}+C M^{1-\theta} \mathcal{D}^{\theta}
$$

for $u$ satisfying equation (7) with $\alpha=\frac{3}{4}$ and $u(x, 0)=0$ for $\forall x \in \Omega$. Here $M$ and $\mathcal{D}$ are defined as follows

$$
\begin{aligned}
& M:=\left\|D_{t}^{\frac{1}{2}} u\right\|_{H^{1,0}(Q)}, \\
& \mathcal{D}:=\sum_{j=-1}^{2}\left\|D_{t}^{\frac{j}{4}} F\right\|_{L^{2}(Q)}+\left\|D_{t}^{\frac{1}{2}} u\right\|_{H^{1}(\Gamma \times(0, T))}+\left\|\partial_{\nu}\left(D_{t}^{\frac{1}{2}} u\right)\right\|_{L^{2}(\Gamma \times(0, T))} .
\end{aligned}
$$

Remark 1.1. Here the generic constant $C$ and constant $\theta$ depend on the choice of $\Omega_{0}, \epsilon$ and the coefficients of the equation (7).

Now on the basis of the above Carleman estimate, let us turn to considering another application: inverse source problem for the equation (7) where the source term is in the form of $F(x, t)=R(x, t) f(x)$. Here again we consider the critical case:

$$
(L u)(x, t)=\partial_{t} u+q(x) \partial_{t}^{\frac{3}{4}} u-\Delta u+B(x) \cdot \nabla u+c(x) u=R(x, t) f(x) \quad \text { in } Q,
$$

where $B(x):=\left(b_{1}(x), \cdots, b_{n}(x)\right)$.

Problem 1 (Inverse source problem). Fix an observation time $t_{0} \in(0, T)$. We intend to determine the spatially varying factor $f$ for given $R$ by measuring the data on some sub-boundary and the value of the solution $u$ at $t=t_{0}$.

The measurements are as the same type as that in the case of a heat equation (See, e.g., 18 for a similar inverse source problem to heat equation). In our problem, we deal with a parabolic equation with some lower-order time-fractional derivative. The idea is to put the fractional derivative term into source term and give some suitable estimates. We have the following conditional stability result on a level set $\Omega_{\epsilon}:=\{x \in \Omega: d(x)>\epsilon\}$ for any $\epsilon>0$.

Theorem 5. Assume that $R \in L^{\infty}(Q)$ with condition $R(\cdot, 0)=0$ in $\Omega$ and $R$ satisfies

$$
R\left(\cdot, t_{0}\right) \neq 0 \quad \text { on } \bar{\Omega}, \quad D_{t}^{\frac{1}{2}} R \in L^{\infty}(Q) .
$$


Then for any $\epsilon>0$ there exist constants $C>0$ and $\theta \in(0,1)$ such that

$$
\|f\|_{L^{2}\left(\Omega_{4 \epsilon}\right)} \leq C \mathcal{D}+C M^{1-\theta} \mathcal{D}^{\theta}
$$

for all $u$ smooth enough and satisfying the equation (13) with

$$
u(\cdot, 0)=\partial_{t} u(\cdot, 0)=0 \quad \text { in } \Omega .
$$

Here by $M$ and $\mathcal{D}$ we denote a priori bound and measurements as follows

$$
\begin{aligned}
& M:=\|f\|_{L^{2}(\Omega)}+\left\|D_{t}^{\frac{3}{2}} u\right\|_{H^{1,0}(Q)}, \\
& \mathcal{D}:=\left\|u\left(x, t_{0}\right)\right\|_{H^{2}(\Omega)}+\left\|D_{t}^{\frac{3}{2}} u\right\|_{H^{1}(\Gamma \times(0, T))}+\left\|D_{t}^{\frac{3}{2}}\left(\partial_{\nu} u\right)\right\|_{L^{2}(\Gamma \times(0, T))},
\end{aligned}
$$

where the generic constant $C$ and constant $\theta$ depend on $\epsilon$ and the coefficients in the equation (13).

To the best knowledge of the authors, most of the existing literatures are focused on the uniqueness of the inverse problems for the time-fractional diffusion equation, see, e.g., [4, 7], [10, 11, [19] and the references therein. This is a first attempt to attack the stability of the inverse source problem (Problem 1) for the time-fractional advection-diffusion equation. Moreover, due to our methods, it is necessary to assume both solution and the time derivative of the solution vanish at the initial time although the Carleman estimate Theorem 3 used for deriving Theorem $[5$ holds true only provided the homogeneous initial value.

The rest of this paper is organized in three sections. In Section 2, by regarding the fractional-order terms as non-homogeneous terms and applying the Carleman estimate for the parabolic equations, we will give a proof for Theorem 1 in the case of the highest fractional order is strictly less than half, and then as a direct application, the conditional stability of a lateral Cauchy problem for the equation (2) stated in Theorem 2 will be established. In Section 3, we first finish the proof for Theorem 3 with a regular weight function which is usually used dealing with the problems in the parabolic equations, and on the basis of the Carleman type estimate in Theorem 3, we will show that the solution continuously depends on Cauchy data and source term. Finally, concluding remarks are given in Section 4

\section{Carleman estimate for the sub-diffusion and its applications}

In this section, we investigate the equation (2) with fractional order $\alpha_{1}<\frac{1}{2}$. We point out that the equation (2) can be regarded a parabolic type equation if we regard the lower fractional order terms as new non-homogeneous terms. Therefore, it is expected to employ the Carleman estimate for the parabolic equations to derive the Carleman estimate for our equation, which is the key idea in this section. Owing to this treatment, in Section 2.1 we will give the proof of Theorem 1 while Theorem 2 will be proved as an application in Section 2.2

\subsection{Carleman estimate for the sub-diffusion}

In this subsection, recalling the notations $d \in C^{2}(\bar{\Omega})$ and $|\nabla d| \neq 0$ on $\bar{\Omega}$, and the function $\psi_{1}=$ $d(x)-\beta t^{2-2 \alpha_{1}}$ with $\beta>0$, we follow the arguments on pp.9-19 of the survey paper [18] to prove the Carleman estimate (5). We use the same notations, where we must modify locally because our choice of the time dependency of $\psi_{1}$ is different.

Proof of Theorem 1. It is sufficient for us to discuss the derivation of a Carleman estimate for $L_{0}=$ $\partial_{t}-\sum_{i, j=1}^{n} a_{i j}(x, t) \partial_{i} \partial_{j}$ with the new weight function $\varphi_{1}=\mathrm{e}^{\lambda \psi_{1}}$. Namely

$$
\begin{aligned}
& \int_{D}\left\{\frac{1}{s \varphi_{1}}\left|\partial_{t} u\right|^{2}+s \lambda^{2} \varphi_{1}|\nabla u|^{2}+s^{3} \lambda^{4} \varphi_{1}^{3} u^{2}\right\} \mathrm{e}^{2 s \varphi_{1}} \mathrm{~d} x \mathrm{~d} t \\
\leq & C \int_{D}\left|L_{0} u\right|^{2} \mathrm{e}^{2 s \varphi_{1}} \mathrm{~d} x \mathrm{~d} t+\mathrm{e}^{C(\lambda) s} \int_{\partial D}\left(|\nabla u|^{2}+|u|^{2}\right) \mathrm{d} S \mathrm{~d} t+\mathrm{e}^{C(\lambda) s} \int_{\partial D \backslash \Sigma_{0}}\left|\partial_{t} u\right|^{2} \mathrm{~d} S \mathrm{~d} t
\end{aligned}
$$


for all $s \geq s_{0}$ and all $u \in H^{2,1}(D)$.

We note

$$
\sigma(x, t)=\sum_{i, j=1}^{n} a_{i j}(x, t)\left(\partial_{i} d\right) \partial_{j} d, \quad(x, t) \in \bar{Q}
$$

and

$$
w(x, t)=\mathrm{e}^{s \varphi_{1}(x, t)} u(x, t)
$$

and

$$
\begin{aligned}
P w=\mathrm{e}^{s \varphi_{1}} L_{0}\left(\mathrm{e}^{-s \varphi_{1}} w\right)= & \partial_{t} w-\sum_{i, j=1}^{n} a_{i j} \partial_{j} \partial_{j} w+2 s \lambda \varphi_{1} \sum_{i, j=1}^{n} a_{i j}\left(\partial_{i} d\right) \partial_{j} w \\
& -s^{2} \lambda^{2} \varphi_{1}^{2} \sigma w+s \lambda^{2} \varphi_{1} \sigma w+s \lambda \varphi_{1} w \sum_{i, j=1}^{n} a_{i j} \partial_{i} \partial_{j} d-s \lambda \varphi_{1} w\left(\partial_{t} \psi_{1}\right) .
\end{aligned}
$$

Now we introduce a new operator $P_{3}$ which is defined by

$$
P_{3} w:=P w+\left(s \lambda^{2} \varphi_{1} \sigma-s \lambda \varphi_{1} \sum_{i, j=1}^{n} a_{i j} \partial_{i} \partial_{j} d+s \lambda \varphi_{1}\left(\partial_{t} \psi_{1}\right)\right) w,
$$

and we decompose $P_{3}$ into the parts $P_{1}$ and $P_{2}$,

$$
P_{3} w=P_{1} w+P_{2} w,
$$

where

$$
P_{1} w=-\sum_{i, j=1}^{n} a_{i j} \partial_{j} \partial_{j} w-s^{2} \lambda^{2} \varphi_{1}^{2} \sigma w
$$

and

$$
P_{2} w=\partial_{t} w+2 s \lambda \varphi_{1} \sum_{i, j=1}^{n} a_{i j}\left(\partial_{i} d\right) \partial_{j} w+2 s \lambda^{2} \varphi_{1} \sigma w .
$$

From the above notations for $P, P_{1}, P_{2}, P_{3}$, it follows that

$$
\begin{aligned}
& \left\|\mathrm{e}^{s \varphi_{1}} L_{0} u+\left(s \lambda^{2} \varphi_{1} \sigma-s \lambda \varphi_{1} \sum_{i, j=1}^{n} a_{i j} \partial_{i} \partial_{j} d+s \lambda \varphi_{1}\left(\partial_{t} \psi_{1}\right)\right) w\right\|_{L^{2}(D)}^{2} \\
= & \left\|P_{1} w+P_{2} w\right\|_{L^{2}(D)}^{2} \geq 2 \int_{D}\left(P_{1} w\right)\left(P_{2} w\right) \mathrm{d} x \mathrm{~d} t+\left\|P_{2} w\right\|_{L^{2}(D)}^{2} .
\end{aligned}
$$

We will estimate $\int_{D}\left|P_{2} w\right|^{2}+2\left(P_{1} w\right)\left(P_{2} w\right) \mathrm{d} x \mathrm{~d} t$ from below. Firstly, we have

$$
\begin{aligned}
\int_{D}\left(P_{1} w\right)\left(P_{2} w\right) \mathrm{d} x \mathrm{~d} t= & -\sum_{i, j=1}^{n} \int_{D} a_{i j}\left(\partial_{i} \partial_{j} w\right) \partial_{t} w \mathrm{~d} x \mathrm{~d} t-\sum_{i, j=1}^{n} \int_{D} a_{i j}\left(\partial_{i} \partial_{j} w\right) 2 s \lambda \varphi_{1} \sum_{k, l=1}^{n} a_{k l}\left(\partial_{k} d\right) \partial_{l} w \mathrm{~d} x \mathrm{~d} t \\
& -\sum_{i, j=1}^{n} \int_{D} a_{i j}\left(\partial_{i} \partial_{j} w\right) 2 s \lambda^{2} \varphi_{1} \sigma w \mathrm{~d} x \mathrm{~d} t-\int_{D} s^{2} \lambda^{2} \varphi_{1}^{2} \sigma w \partial_{t} w \mathrm{~d} x \mathrm{~d} t \\
& -\int_{D} 2 s^{3} \lambda^{3} \varphi_{1}^{3} \sigma w \sum_{i, j=1}^{n} a_{i j}\left(\partial_{i} d\right) \partial_{j} w \mathrm{~d} x \mathrm{~d} t-\int_{D} 2 s^{3} \lambda^{4} \varphi_{1}^{3} \sigma^{2} w^{2} \mathrm{~d} x \mathrm{~d} t=: \sum_{k=1}^{6} J_{k}
\end{aligned}
$$

Now, applying the integration by parts and the symmetry of $\left\{a_{i j}\right\}: a_{i j}=a_{j i}$, we give the estimates of $J_{k}$, $k=1, \cdots, 6$ separately.

$$
J_{1}=-\sum_{i, j=1}^{n} \int_{D} a_{i j}\left(\partial_{i} \partial_{j} w\right) \partial_{t} w \mathrm{~d} x \mathrm{~d} t
$$




$$
=\sum_{i, j=1}^{n} \int_{D}\left(\partial_{i} a_{i j}\right)\left(\partial_{j} w\right) \partial_{t} w \mathrm{~d} x \mathrm{~d} t+\sum_{i, j=1}^{n} \int_{D} a_{i j}\left(\partial_{j} w\right) \partial_{i} \partial_{t} w \mathrm{~d} x \mathrm{~d} t-\sum_{i, j=1}^{n} \int_{\partial D} a_{i j}\left(\partial_{j} w\right) \nu_{i} \partial_{t} w \mathrm{~d} \Sigma
$$

Here and henceforth $\nu:=\left(\nu_{1}, \cdots, \nu_{n}, \nu_{n+1}\right)$ denotes the unit normal exterior with respect to the boundary $\partial D$ of $D$. In particular, $\nu_{n+1}$ is the component in the time direction. By noting $\nu_{i}=0, \forall i=1, \ldots, n$ on $\Sigma_{0}$, then integration by parts yields

$$
\begin{aligned}
J_{1}= & \sum_{i, j=1}^{n} \int_{D}\left(\partial_{i} a_{i j}\right)\left(\partial_{j} w\right) \partial_{t} w \mathrm{~d} x \mathrm{~d} t+\frac{1}{2} \sum_{i, j=1}^{n} \int_{D} a_{i j} \partial_{t}\left(\left(\partial_{i} w\right) \partial_{j} w\right) \mathrm{d} x \mathrm{~d} t \\
& -\sum_{i, j=1}^{n} \int_{\partial D \backslash \Sigma_{0}} a_{i j}\left(\partial_{j} w\right) \nu_{i} \partial_{t} w \mathrm{~d} \Sigma \\
= & \sum_{i, j=1}^{n} \int_{D}\left(\partial_{i} a_{i j}\right)\left(\partial_{j} w\right) \partial_{t} w \mathrm{~d} x \mathrm{~d} t-\frac{1}{2} \sum_{i, j=1}^{n} \int_{D}\left(\partial_{t} a_{i j}\right)\left(\partial_{i} w\right) \partial_{j} w \mathrm{~d} x \mathrm{~d} t \\
& +\frac{1}{2} \sum_{i, j=1}^{n} \int_{\partial D} a_{i j}\left(\partial_{i} w\right)\left(\partial_{j} w\right) \nu_{n+1} \mathrm{~d} \Sigma-\sum_{i, j=1}^{n} \int_{\partial D \backslash \Sigma_{0}} a_{i j}\left(\partial_{j} w\right) \nu_{i} \partial_{t} w \mathrm{~d} \Sigma .
\end{aligned}
$$

Thus

$$
\begin{aligned}
\left|J_{1}\right| & \leq C \int_{D}|\nabla w|\left|\partial_{t} w\right| \mathrm{d} x \mathrm{~d} t+C \int_{D}|\nabla w|^{2} \mathrm{~d} x \mathrm{~d} t+C \int_{\partial D}|\nabla w|^{2} \mathrm{~d} \Sigma+C \int_{\partial D \backslash \Sigma_{0}}|\nabla w|\left|\partial_{t} w\right| \mathrm{d} \Sigma \\
& \leq C \int_{D}|\nabla w|\left|\partial_{t} w\right| \mathrm{d} x \mathrm{~d} t+C \int_{D}|\nabla w|^{2} \mathrm{~d} x \mathrm{~d} t+C \int_{\partial D}|\nabla w|^{2} \mathrm{~d} \Sigma+C \int_{\partial D \backslash \Sigma_{0}}\left|\partial_{t} w\right|^{2} \mathrm{~d} \Sigma .
\end{aligned}
$$

Since the Cauchy-Schwarz inequality implies that

$$
|\nabla w|\left|\partial_{t} w\right|=s^{\frac{1}{2}} \lambda^{\frac{1}{2}} \varphi_{1}^{\frac{1}{2}}|\nabla w| s^{-\frac{1}{2}} \lambda^{-\frac{1}{2}} \varphi_{1}^{-\frac{1}{2}}\left|\partial_{t} w\right| \leq \frac{1}{2} s \lambda \varphi_{1}|\nabla w|^{2}+\frac{1}{2} \frac{1}{s \lambda \varphi_{1}}\left|\partial_{t} w\right|^{2},
$$

we have

$$
\begin{aligned}
\left|J_{1}\right| \leq & C \int_{D} \frac{1}{s \lambda \varphi_{1}}\left|\partial_{t} w\right|^{2} \mathrm{~d} x \mathrm{~d} t+C \int_{D} s \lambda \varphi_{1}|\nabla w|^{2} \mathrm{~d} x \mathrm{~d} t \\
& +C \int_{\partial D}|\nabla w|^{2} \mathrm{~d} \Sigma+C \int_{\partial D \backslash \Sigma_{0}}\left|\partial_{t} w\right|^{2} \mathrm{~d} \Sigma .
\end{aligned}
$$

Next similar to the argument on pp. 12-13 in [18, we have

$$
\begin{aligned}
J_{2}= & -\sum_{i, j=1}^{n} \sum_{k, l=1}^{n} \int_{D} 2 s \lambda \varphi_{1} a_{i j} a_{k l}\left(\partial_{k} d\right)\left(\partial_{l} w\right) \partial_{i} \partial_{j} w \mathrm{~d} x \mathrm{~d} t \\
= & 2 s \lambda \int_{D} \sum_{i, j=1}^{n} \sum_{k, l=1}^{n} \lambda\left(\partial_{i} d\right) \varphi_{1} a_{i j} a_{k l}\left(\partial_{k} d\right)\left(\partial_{l} w\right) \partial_{j} w \mathrm{~d} x \mathrm{~d} t \\
& +2 s \lambda \int_{D} \sum_{i, j=1}^{n} \sum_{k, l=1}^{n} \varphi_{1} \partial_{i}\left(a_{i j} a_{k l} \partial_{k} d\right)\left(\partial_{l} w\right) \partial_{j} w \mathrm{~d} x \mathrm{~d} t \\
& +2 s \lambda \int_{D} \sum_{i, j=1}^{n} \sum_{k, l=1}^{n} \varphi_{1} a_{i j} a_{k l}\left(\partial_{k} d\right)\left(\partial_{i} \partial_{l} w\right) \partial_{j} w \mathrm{~d} x \mathrm{~d} t \\
& -2 s \lambda \int_{\partial D} \sum_{i, j=1}^{n} \sum_{k, l=1}^{n} \varphi_{1} a_{i j} a_{k l}\left(\partial_{k} d\right)\left(\partial_{l} w\right)\left(\partial_{j} w\right) \nu_{i} \mathrm{~d} \Sigma .
\end{aligned}
$$


We have

$$
(\text { first term })=2 s \lambda^{2} \int_{D} \varphi_{1}\left|\sum_{i, j=1}^{n} a_{i j}\left(\partial_{i} d\right) \partial_{j} w\right|^{2} \mathrm{~d} x \mathrm{~d} t \geq 0
$$

and

$$
\begin{aligned}
(\text { third term })= & s \lambda \int_{D} \varphi_{1} \sum_{i, j=1}^{n} \sum_{k, l=1}^{n} a_{i j} a_{k l}\left(\partial_{k} d\right) \partial_{l}\left(\left(\partial_{i} w\right)\left(\partial_{j} w\right)\right) \mathrm{d} x \mathrm{~d} t \\
= & s \lambda \int_{\partial D} \varphi_{1} \sum_{i, j=1}^{n} \sum_{k, l=1}^{n} a_{i j} a_{k l}\left(\partial_{k} d\right)\left(\partial_{i} w\right)\left(\partial_{j} w\right) \nu_{l} \mathrm{~d} \Sigma \\
& -s \lambda^{2} \int_{D} \varphi_{1} \sum_{i, j=1}^{n} \sigma a_{i j}\left(\partial_{i} w\right) \partial_{j} w \mathrm{~d} x \mathrm{~d} t \\
& -s \lambda \int_{D} \varphi_{1} \sum_{i, j=1}^{n} \sum_{k, l=1}^{n} \partial_{l}\left(a_{i j} a_{k l}\left(\partial_{k} d\right)\right)\left(\partial_{i} w\right) \partial_{j} w \mathrm{~d} x \mathrm{~d} t
\end{aligned}
$$

which imply

$$
\begin{aligned}
J_{2} \geq & -\int_{D} s \lambda^{2} \varphi_{1} \sigma \sum_{i, j=1}^{n} a_{i j}\left(\partial_{i} w\right) \partial_{j} w \mathrm{~d} x \mathrm{~d} t-C \int_{D} s \lambda \varphi_{1}|\nabla w|^{2} \mathrm{~d} x \mathrm{~d} t-C \int_{\partial D} s \lambda \varphi_{1}|\nabla w|^{2} \mathrm{~d} \Sigma . \\
J_{3}= & 2 \int_{D} s \lambda^{2} \varphi_{1} \sigma \sum_{i, j=1}^{n} a_{i j}\left(\partial_{i} w\right) \partial_{j} w \mathrm{~d} x \mathrm{~d} t+2 \int_{D} s \lambda^{2} \sum_{i, j=1}^{n} \partial_{i}\left(\varphi_{1} \sigma a_{i j}\right) w \partial_{j} w \mathrm{~d} x \mathrm{~d} t \\
& -2 \int_{\partial D} s \lambda^{2} \varphi_{1} \sigma \sum_{i, j=1}^{n} a_{i j} w\left(\partial_{j} w\right) \nu_{i} \mathrm{~d} \Sigma \\
\geq & 2 \int_{D} s \lambda^{2} \varphi_{1} \sigma \sum_{i, j=1}^{n} a_{i j}\left(\partial_{i} w\right) \partial_{j} w \mathrm{~d} x \mathrm{~d} t-C \int_{D} s \lambda^{3} \varphi_{1}|\nabla w \| w| \mathrm{d} x \mathrm{~d} t-C \int_{\partial D} s \lambda^{2} \varphi_{1}|\nabla w||w| \mathrm{d} \Sigma .
\end{aligned}
$$

By

$$
s \lambda^{3} \varphi_{1}|\nabla w||w|=\left(s \lambda^{2} \varphi_{1}|w|\right)(\lambda|\nabla w|) \leq \frac{1}{2} s^{2} \lambda^{4} \varphi_{1}^{2} w^{2}+\frac{1}{2} \lambda^{2}|\nabla w|^{2}
$$

and

$$
s \lambda^{2} \varphi_{1}|\nabla w||w|=\left(s \lambda^{\frac{3}{2}} \varphi_{1}|w|\right)\left(\lambda^{\frac{1}{2}}|\nabla w|\right) \leq \frac{1}{2} s^{2} \lambda^{3} \varphi_{1}^{2} w^{2}+\frac{1}{2} \lambda|\nabla w|^{2},
$$

we have

$$
\begin{aligned}
& J_{3} \geq 2 \int_{D} s \lambda^{2} \varphi_{1} \sigma \sum_{i, j=1}^{n} a_{i j}\left(\partial_{i} w\right) \partial_{j} w \mathrm{~d} x \mathrm{~d} t-C \int_{D} s^{2} \lambda^{4} \varphi_{1}^{2} w^{2} \mathrm{~d} x \mathrm{~d} t-C \int_{D} \lambda^{2}|\nabla w|^{2} \mathrm{~d} x \mathrm{~d} t \\
& \quad-C \int_{\partial D} \lambda|\nabla w|^{2} \mathrm{~d} \Sigma-C \int_{\partial D} s^{2} \lambda^{3} \varphi_{1}^{2} w^{2} \mathrm{~d} \Sigma . \\
& \left|J_{4}\right|=\left|-\frac{1}{2} \int_{D} s^{2} \lambda^{2} \varphi_{1}^{2} \sigma \partial_{t}\left(w^{2}\right) \mathrm{d} x \mathrm{~d} t\right| \\
& =\left|\int_{D} s^{2} \lambda^{3} \varphi_{1}^{2} \beta\left(2 \alpha_{1}-2\right) t^{1-2 \alpha_{1}} \sigma w^{2} \mathrm{~d} x \mathrm{~d} t+\frac{1}{2} \int_{D} s^{2} \lambda^{2} \varphi_{1}^{2}\left(\partial_{t} \sigma\right) w^{2} \mathrm{~d} x \mathrm{~d} t-\frac{1}{2} \int_{\partial D} s^{2} \lambda^{2} \varphi_{1}^{2} \sigma w^{2} \nu_{n+1} \mathrm{~d} \Sigma\right| \\
& \leq C \int_{D} s^{2} \lambda^{3} \varphi_{1}^{2} w^{2} \mathrm{~d} x \mathrm{~d} t+C \int_{\partial D} s^{2} \lambda^{2} \varphi_{1}^{2} w^{2} \mathrm{~d} \Sigma .
\end{aligned}
$$




$$
\begin{aligned}
& J_{5}=-\int_{D} s^{3} \lambda^{3} \varphi_{1}^{3} \sum_{i, j=1}^{n} \sigma a_{i j}\left(\partial_{i} d\right) \partial_{j}\left(w^{2}\right) \mathrm{d} x \mathrm{~d} t \\
&= 3 \int_{D} s^{3} \lambda^{4} \varphi_{1}^{3} \sigma^{2} w^{2} \mathrm{~d} x \mathrm{~d} t+\int_{D} s^{3} \lambda^{3} \varphi_{1}^{3} \sum_{i, j=1}^{n} \partial_{j}\left(\sigma a_{i j} \partial_{i} d\right) w^{2} \mathrm{~d} x \mathrm{~d} t \\
&-\int_{\partial D} s^{3} \lambda^{3} \varphi_{1}^{3} \sum_{i, j=1}^{n} \sigma a_{i j}\left(\partial_{i} d\right) w^{2} \nu_{j} \mathrm{~d} \Sigma \\
& \geq 3 \int_{D} s^{3} \lambda^{4} \varphi_{1}^{3} \sigma^{2} w^{2} \mathrm{~d} x \mathrm{~d} t-C \int_{D} s^{3} \lambda^{3} \varphi_{1}^{3} w^{2} \mathrm{~d} x \mathrm{~d} t-C \int_{\partial D} s^{3} \lambda^{3} \varphi_{1}^{3} w^{2} \mathrm{~d} \Sigma . \\
& J_{6}=-\int_{D} 2 s^{3} \lambda^{4} \varphi_{1}^{3} \sigma^{2} w^{2} \mathrm{~d} x \mathrm{~d} t .
\end{aligned}
$$

By the definition of $P_{2}$, we have

$$
\begin{aligned}
\epsilon \int_{D} \frac{1}{s \varphi_{1}}\left|\partial_{t} w\right|^{2} \mathrm{~d} x \mathrm{~d} t & =\epsilon \int_{D} \frac{1}{s \varphi_{1}}\left|P_{2} w-2 s \lambda \varphi_{1} \sum_{i, j=1}^{n} a_{i j}\left(\partial_{i} d\right) \partial_{j} w-2 s \lambda^{2} \varphi_{1} \sigma w\right|^{2} \mathrm{~d} x \mathrm{~d} t \\
& \leq C \int_{D}\left|P_{2} w\right|^{2} \mathrm{~d} x \mathrm{~d} t+C \epsilon \int_{D} s \lambda^{2} \varphi_{1}|\nabla w|^{2} \mathrm{~d} x \mathrm{~d} t+C \epsilon \int_{D} s \lambda^{4} \varphi_{1} w^{2} \mathrm{~d} x \mathrm{~d} t
\end{aligned}
$$

By summing up all the above estimates for $J_{k}, k=1, \cdots, 6$, we find

$$
\begin{aligned}
& \int_{D} s^{3} \lambda^{4} \varphi_{1}^{3} \sigma^{2} w^{2} \mathrm{~d} x \mathrm{~d} t+\int_{D} s \lambda^{2} \varphi_{1} \sigma \sum_{i, j=1}^{n} a_{i j}\left(\partial_{i} w\right) \partial_{j} w \mathrm{~d} x \mathrm{~d} t+\left(\epsilon-\frac{C}{\lambda}\right) \int_{D} \frac{1}{s \varphi_{1}}\left|\partial_{t} w\right|^{2} \mathrm{~d} x \mathrm{~d} t \\
\leq & C \int_{D}\left|L_{0} u\right|^{2} e^{2 s \varphi_{1}} \mathrm{~d} x \mathrm{~d} t+C \int_{D}\left(s \lambda \varphi_{1}+\epsilon s \lambda^{2} \varphi_{1}+\lambda^{2}\right)|\nabla w|^{2} \mathrm{~d} x \mathrm{~d} t+C \int_{D}\left(s^{3} \lambda^{3} \varphi_{1}^{3}+s^{2} \lambda^{4} \varphi_{1}^{2}\right) w^{2} \mathrm{~d} x \mathrm{~d} t \\
& +C \int_{\partial D}\left(s \lambda \varphi_{1}|\nabla w|^{2}+s^{3} \lambda^{3} \varphi_{1}^{3} w^{2}\right) \mathrm{d} \Sigma+C \int_{\partial D \backslash \Sigma_{0}}\left|\partial_{t} w\right|^{2} \mathrm{~d} \Sigma,
\end{aligned}
$$

which combined with the ellipticity of $a_{i j}$ and $\sigma_{0}:=\inf _{(x, t) \in Q} \sigma(x, t)>0$ yields that

$$
\begin{aligned}
& \int_{D} s^{3} \lambda^{4} \varphi_{1}^{3} \sigma_{0}^{2} w^{2} d x d t+\int_{D}\left(\sigma_{0} \rho-C \epsilon\right) s \lambda^{2} \varphi_{1}|\nabla w|^{2} \mathrm{~d} x \mathrm{~d} t+\left(\epsilon-\frac{C}{\lambda}\right) \int_{D} \frac{1}{s \varphi_{1}}\left|\partial_{t} w\right|^{2} \mathrm{~d} x \mathrm{~d} t \\
\leq & C \int_{D}\left|L_{0} u\right|^{2} e^{2 s \varphi_{1}} \mathrm{~d} x \mathrm{~d} t+C \int_{D}\left(s^{3} \lambda^{3} \varphi_{1}^{3}+s^{2} \lambda^{4} \varphi_{1}^{2}\right) w^{2} \mathrm{~d} x \mathrm{~d} t+C \int_{D}\left(s \lambda \varphi_{1}+\lambda^{2}\right)|\nabla w|^{2} \mathrm{~d} x \mathrm{~d} t \\
& +\int_{\partial D}\left(s \lambda \varphi_{1}|\nabla w|^{2}+s^{2} \lambda^{3} \varphi_{1}^{2} w^{2}\right) \mathrm{d} \Sigma+\int_{\partial D \backslash \Sigma_{0}}\left|\partial_{t} w\right|^{2} \mathrm{~d} \Sigma .
\end{aligned}
$$

Thus choosing $\epsilon>0$ small, and choosing $\lambda$ and then $s$ large, we can absorb terms suitably to obtain

$$
\begin{aligned}
& \int_{D}\left\{\frac{1}{s \varphi_{1}}\left|\partial_{t} w\right|^{2}+s \lambda^{2} \varphi_{1}|\nabla w|^{2}+s^{3} \lambda^{4} \varphi_{1}^{3} w^{2}\right\} \mathrm{d} x \mathrm{~d} t \\
\leq & C \int_{D}\left|L_{0} u\right|^{2} \mathrm{e}^{2 s \varphi_{1}} \mathrm{~d} x \mathrm{~d} t+\int_{\partial D}\left(s \lambda \varphi_{1}|\nabla w|^{2}+s^{2} \lambda^{3} \varphi_{1}^{2} w^{2}\right) \mathrm{d} \Sigma+\int_{\partial D \backslash \Sigma_{0}}\left|\partial_{t} w\right|^{2} \mathrm{~d} \Sigma .
\end{aligned}
$$

Noting $w=u \mathrm{e}^{s \varphi_{1}}$, we have

$$
\begin{aligned}
& \int_{D}\left\{\frac{1}{s \varphi_{1}}\left|\partial_{t} u\right|^{2}+s \lambda^{2} \varphi_{1}|\nabla u|^{2}+s^{3} \lambda^{4} \varphi_{1}^{3} u^{2}\right\} \mathrm{e}^{2 s \varphi_{1}} \mathrm{~d} x \mathrm{~d} t \\
\leq & C \int_{D}\left|L_{0} u\right|^{2} \mathrm{e}^{2 s \varphi_{1}} \mathrm{~d} x \mathrm{~d} t+C(\lambda) \mathrm{e}^{C(\lambda) s} \int_{\partial D}\left(|\nabla u|^{2}+u^{2}\right) \mathrm{d} \Sigma+C(\lambda) \mathrm{e}^{C(\lambda) s} \int_{\partial D \backslash \Sigma_{0}}\left|\partial_{t} u\right|^{2} \mathrm{~d} \Sigma,
\end{aligned}
$$

which completes the proof of the theorem. 


\subsection{Application to a lateral Cauchy problem for the sub-diffusion}

In this section, we will give a proof of Theorem 2 To prove this, we follow the usual argument used in analyzing the lateral Cauchy problem for the parabolic equations, that is, we use the Carleman estimate derived in Section 2.1. The first problem which we have to overcome is to evaluate the fractional derivative by the first order time-derivative under some suitable norm. Namely, the following lemma holds.

Lemma 2.1. Let $T>0$ and $0<\alpha \leq \alpha_{1}<\frac{1}{2}$ be given constants, then the following inequality

$$
\int_{\mathcal{C}_{1}}\left|\partial_{t}^{\alpha} u\right|^{2} \mathrm{e}^{2 s \varphi_{1}} \mathrm{~d} x \mathrm{~d} t \leq C \int_{\mathcal{C}_{2}} \frac{1}{s \lambda \varphi_{1}}\left|\partial_{t} u\right|^{2} \mathrm{e}^{2 s \varphi_{1}} \mathrm{~d} x \mathrm{~d} t
$$

holds true for all $u \in H^{2,1}(Q)$, where $\varphi_{1}=\mathrm{e}^{\lambda \psi_{1}}$ with $\psi_{1}(x, t)=d(x)-\beta t^{2-2 \alpha_{1}}$, and $\mathcal{C}_{i}:=\{(x, t) ; x \in \bar{\Omega}, t>$ $\left.0, \varphi_{1}(x, t)>c_{i}\right\}, i=1,2$, and $c_{i}$ are positive constants such that $c_{2}<c_{1}$.

Proof. We choose a nonnegative function $\Phi \in L^{\infty}\left(\mathbb{R}^{n+1}\right)$ such that $\operatorname{supp} \Phi \subset \mathcal{C}_{2}$ and $\Phi \equiv 1$ in $\mathcal{C}_{1}$. Thus we have

$$
\int_{\mathcal{C}_{1}}\left|\partial_{t}^{\alpha} u\right|^{2} \mathrm{e}^{2 s \varphi_{1}} \mathrm{~d} x \mathrm{~d} t=\int_{\mathcal{C}_{1}} \Phi(x, t)\left|\partial_{t}^{\alpha} u\right|^{2} \mathrm{e}^{2 s \varphi_{1}} \mathrm{~d} x \mathrm{~d} t,
$$

which combined with the definition of the Caputo derivative implies that

$$
\int_{\mathcal{C}_{1}} \Phi(x, t)\left|\partial_{t}^{\alpha} u\right|^{2} \mathrm{e}^{2 s \varphi_{1}} \mathrm{~d} x \mathrm{~d} t=\int_{\mathcal{C}_{1}}\left|\frac{\Phi^{\frac{1}{2}}(x, t)}{\Gamma(1-\alpha)} \int_{0}^{t}(t-r)^{-\alpha} \partial_{r} u(x, r) \mathrm{d} r\right|^{2} \mathrm{e}^{2 s \varphi_{1}} \mathrm{~d} x \mathrm{~d} t .
$$

Moreover, since for any fixed $x \in \Omega, \varphi_{1}(x, t)$ is decreasing with respect to the variable $t>0$, we see that $\varphi_{1}(x, t) \leq \varphi_{1}(x, r)$ for any $x \in \Omega$ and $0<r \leq t$, so that $(x, t) \in \mathcal{C}_{1}$ implies that $(x, r) \in \mathcal{C}_{1}$ for $0<r \leq t$, hence that $\Phi(x, r)=1$ if $(x, t) \in \mathcal{C}_{1}$ and $0<r<t$, finally we have

$$
\begin{aligned}
\int_{\mathcal{C}_{1}} \Phi(x, t)\left|\partial_{t}^{\alpha} u\right|^{2} \mathrm{e}^{2 s \varphi_{1}} \mathrm{~d} x \mathrm{~d} t & =\int_{\mathcal{C}_{1}}\left|\frac{1}{\Gamma(1-\alpha)} \int_{0}^{t}(t-r)^{-\alpha} \Phi^{\frac{1}{2}}(x, r) \partial_{r} u(x, r) \mathrm{d} r\right|^{2} \mathrm{e}^{2 s \varphi_{1}} \mathrm{~d} x \mathrm{~d} t \\
& \leq \int_{Q}\left|\frac{1}{\Gamma(1-\alpha)} \int_{0}^{t}(t-r)^{-\alpha} \Phi^{\frac{1}{2}}(x, r) \partial_{r} u(x, r) \mathrm{d} r\right|^{2} \mathrm{e}^{2 s \varphi_{1}} \mathrm{~d} x \mathrm{~d} t .
\end{aligned}
$$

Now by noting that $\varphi_{1}(x, t) \geq c_{0}$, where $c_{0}>0$ is a constant, we have

$$
\partial_{t} \psi_{1}=\beta\left(2 \alpha_{1}-2\right) t^{1-2 \alpha_{1}}, \quad \partial_{t} \varphi_{1}=\lambda\left(\partial_{t} \psi_{1}\right) \varphi_{1}=\left(2 \alpha_{1}-2\right) \beta \lambda \varphi_{1} t^{1-2 \alpha_{1}} .
$$

Hence

$$
t^{1-2 \alpha_{1}} e^{2 s \varphi_{1}}=-\frac{1}{4 \beta s \lambda \varphi_{1}\left(1-\alpha_{1}\right)} \partial_{t}\left(e^{2 s \varphi_{1}}\right)
$$

By the Cauchy-Schwarz inequality and (3), we have

$$
\begin{aligned}
& \int_{0}^{T}\left|\int_{0}^{t}(t-r)^{-\alpha} \Phi^{\frac{1}{2}}(x, r) \partial_{r} u(r) \mathrm{d} r\right|^{2} \mathrm{e}^{2 s \varphi_{1}(x, t)} \mathrm{d} t \\
\leq & \int_{0}^{T}\left(\int_{0}^{t}(t-r)^{-2 \alpha} \mathrm{d} r\right)\left(\int_{0}^{t} \Phi(x, r)\left|\partial_{r} u(r)\right|^{2} \mathrm{~d} r\right) \mathrm{e}^{2 s \varphi_{1}(x, t)} \mathrm{d} t \\
= & \frac{1}{1-2 \alpha} \int_{0}^{T} t^{1-2 \alpha}\left(\int_{0}^{t} \Phi(x, r)\left|\partial_{r} u(r)\right|^{2} \mathrm{~d} r\right) \mathrm{e}^{2 s \varphi_{1}(x, t)} \mathrm{d} t
\end{aligned}
$$

Moreover, since $0<\alpha<\alpha_{1}$, we see that

$$
\int_{0}^{T}\left|\int_{0}^{t}(t-r)^{-\alpha} \Phi^{\frac{1}{2}}(x, r) \partial_{r} u(r) \mathrm{d} r\right|^{2} \mathrm{e}^{2 s \varphi_{1}} \mathrm{~d} t \leq \frac{T^{2\left(\alpha_{1}-\alpha\right)}}{1-2 \alpha} \int_{0}^{T} t^{1-2 \alpha_{1}}\left(\int_{0}^{t} \Phi(x, r)\left|\partial_{r} u(r)\right|^{2} \mathrm{~d} r\right) \mathrm{e}^{2 s \varphi_{1}} \mathrm{~d} t .
$$


Now from the formula (18), integration by parts implies

$$
\begin{aligned}
& \int_{0}^{T} t^{1-2 \alpha_{1}}\left(\int_{0}^{t} \Phi(x, r)\left|\partial_{r} u(r)\right|^{2} \mathrm{~d} r\right) \mathrm{e}^{2 s \varphi_{1}(x, t)} \mathrm{d} t \\
= & \left.\frac{1}{1-\alpha_{1}}\left(\frac{-1}{4 \beta s \lambda \varphi_{1}} \int_{0}^{t} \Phi(x, r)\left|\partial_{r} u(r)\right|^{2} \mathrm{~d} r\right) \mathrm{e}^{2 s \varphi_{1}(x, t)}\right|_{t=0} ^{t=T}+\frac{1}{1-\alpha_{1}} \int_{0}^{T} \frac{\Phi(x, t)}{4 \beta s \lambda \varphi_{1}}\left|\partial_{t} u\right|^{2} \mathrm{e}^{2 s \varphi_{1}} \mathrm{~d} t \\
+ & \int_{0}^{T} \frac{t^{1-2 \alpha_{1}}}{2 s \varphi_{1}}\left(\int_{0}^{t} \Phi(x, r)\left|\partial_{r} u(r)\right|^{2} \mathrm{~d} r\right) \mathrm{e}^{2 s \varphi_{1}} \mathrm{~d} t \\
\leq & \frac{1}{1-\alpha_{1}} \int_{0}^{T} \frac{\Phi(x, t)}{4 \beta s \lambda \varphi_{1}}\left|\partial_{t} u\right|^{2} \mathrm{e}^{2 s \varphi_{1}} \mathrm{~d} t+\int_{0}^{T} \frac{t^{1-2 \alpha_{1}}}{2 s \varphi_{1}}\left(\int_{0}^{t} \Phi(x, r)\left|\partial_{r} u(r)\right|^{2} d r\right) \mathrm{e}^{2 s \varphi_{1}} \mathrm{~d} t .
\end{aligned}
$$

The last term on the right-hand side can be absorbed into the left-hand side by choosing $s>0$ large and we have

$$
\int_{0}^{T} t^{1-2 \alpha_{1}}\left(\int_{0}^{t} \Phi(x, r)\left|\partial_{r} u(r)\right|^{2} \mathrm{~d} r\right) \mathrm{e}^{2 s \varphi_{1}(x, t)} \mathrm{d} t \leq C \int_{0}^{T} \frac{\Phi(x, t)}{s \lambda \varphi_{1}}\left|\partial_{t} u(t)\right|^{2} \mathrm{e}^{2 s \varphi_{1}} \mathrm{~d} t .
$$

Thus

$$
\int_{Q} t^{1-2 \alpha_{1}}\left(\int_{0}^{t} \Phi(x, r)\left|\partial_{r} u(r)\right|^{2} \mathrm{~d} r\right) \mathrm{e}^{2 s \varphi_{1}(x, t)} \mathrm{d} x \mathrm{~d} t \leq C \int_{Q} \frac{\Phi(x, t)}{s \lambda \varphi_{1}}\left|\partial_{t} u(t)\right|^{2} \mathrm{e}^{2 s \varphi_{1}} \mathrm{~d} x \mathrm{~d} t,
$$

which combined with the fact supp $\Phi \subset \mathcal{C}_{2}$ implies that

$$
\int_{\mathcal{C}_{1}}\left|\partial_{t}^{\alpha} u\right|^{2} \mathrm{e}^{2 s \varphi_{1}} \mathrm{~d} x \mathrm{~d} t \leq C \int_{\mathcal{C}_{2}} \frac{1}{s \lambda \varphi_{1}}\left|\partial_{t} u\right|^{2} \mathrm{e}^{2 s \varphi_{1}} \mathrm{~d} x \mathrm{~d} t,
$$

which completes the proof of the lemma.

Before giving the proof of Theorem 2, we introduce some notations.

For arbitrary given domain $\Omega_{0}$ such that $\overline{\Omega_{0}} \subset \Omega$, similar to Theorem 5.1 in 18 , we will choose a suitable weight function $\psi_{1}(x, t):=d(x)-\beta t^{2-2 \alpha_{1}}$. For this, we first choose a bounded domain $\Omega_{1}$ with smooth boundary such that

$$
\Omega \subsetneq \Omega_{1}, \quad \bar{\Gamma}=\overline{\partial \Omega \cap \Omega_{1}}, \quad \partial \Omega \backslash \Gamma \subset \partial \Omega_{1} .
$$

We then apply Lemma 4.1 in $\left[18\right.$ to obtain $d \in C^{2}\left(\overline{\Omega_{1}}\right)$ satisfying

$$
d(x)>0, x \in \Omega_{1}, \quad d(x)=0, x \in \partial \Omega_{1}, \quad|\nabla d(x)|>0, x \in \bar{\Omega} .
$$

Then we can choose $\beta>0$ such that

$$
\beta\left(\frac{T}{2}\right)^{2-2 \alpha_{1}}<\|d\|_{C\left(\overline{\Omega_{1}}\right)}<\beta T^{2-2 \alpha_{1}} .
$$

Moreover, since $\overline{\Omega_{0}} \subset \Omega_{1}$, we can choose a sufficiently large $N>1$ such that

$$
\Omega_{0} \subset \bar{\Omega} \cap\left\{x \in \Omega_{1} ; d(x)>\frac{4}{N}\|d\|_{C\left(\overline{\Omega_{1}}\right)}\right\} .
$$

We set $\mu_{k}=\exp \left\{\lambda\left(\frac{k}{N}\|d\|_{C\left(\overline{\Omega_{1}}\right)}-\frac{\beta\left(\frac{T}{2}\right)^{2-2 \alpha_{1}}}{N}\right)\right\}$, and $D_{k}:=\left\{(x, t) ; x \in \bar{\Omega}, t>0, \varphi(x, t)>\mu_{k}\right\}, k=$ $1,2,3,4$. Then we can verify from (19) and (20) that

$$
\Omega_{0} \times\left(0, \frac{T}{2 M}\right) \subset D_{4} \subset D_{3} \subset D_{1} \subset \bar{\Omega} \times(0, T),
$$

where $M:=N^{\frac{1}{2-2 \alpha_{1}}}$, and

$$
\partial D_{1} \subset \Sigma_{0} \cup \Sigma_{1} \cup \Sigma_{2}
$$

are valid. Here $\Sigma_{0}=\{(x, 0) ; x \in \bar{\Omega}\}, \Sigma_{1} \subset \Gamma \times(0, T)$ and $\Sigma_{2}=\left\{(x, t) ; x \in \Omega, t>0, \varphi(x, t)=\mu_{1}\right\}$.

Now we are ready to give the proof of our main theorem. 
Proof of Theorem 2. We start from the Cauchy problem

$$
\begin{cases}u(x, t)=g_{0}(x, t) & \text { on } \Gamma \times(0, T] \\ \partial_{\nu_{A}} u(x, t)=g_{1}(x, t) & \text { on } \Gamma \times(0, T]\end{cases}
$$

for the equation (2).

Henceforth $C>0$ denotes generic constants depending on $\lambda$, but independent of $s$ and the choice of $g_{0}$, $g_{1}, u$. For it, we need a cut-off function because we have no data $\partial_{\nu_{A}} u$ on $\partial D \backslash \Gamma \times(0, T)$. Let $\chi \in C^{\infty}\left(\mathbb{R}^{n+1}\right)$ such that $0 \leq \chi \leq 1$ and

$$
\chi(x, t)= \begin{cases}1, & \varphi_{1}(x, t)>\mu_{3}, \\ 0, & \varphi_{1}(x, t)<\mu_{2} .\end{cases}
$$

Setting $v:=\chi u, \widetilde{L}:=L-\sum_{j=1}^{\ell} q_{j} \partial_{t}^{\alpha_{j}}$, and then using Leibniz's formula for the differential of the product we have

$$
\widetilde{L} v=\chi \widetilde{L} u+A_{1} u=\chi L u-\chi \sum_{j=1}^{\ell} q_{j} \partial_{t}^{\alpha_{j}} u+A_{1} u=\chi F-\chi \sum_{j=1}^{\ell} q_{j} \partial_{t}^{\alpha_{j}} u+A_{1} u
$$

Here the last term $A_{1} u$ involves only the linear combination of $\left(\partial_{t} \chi\right) u,\left(\partial_{i} \partial_{j} \chi\right) u,\left(\partial_{i} \chi\right)\left(\partial_{j} u\right)$ and $\left(\partial_{i} \chi\right) u$, $i, j=1, \cdots, n$.

By (22) and (23), we see that $v=|\nabla v|=\partial_{t} v=0$ on $\Sigma_{2}$. Hence using the Carleman estimate in Theorem 1 from $D_{3} \subset D_{1}$ by an argument similar to Theorem 3.2 in [18] in $D_{1}$ to (24), we find

$$
\begin{aligned}
& \int_{D_{3}}\left\{\frac{1}{s \varphi_{1}}\left|\partial_{t} v\right|^{2}+s \lambda^{2} \varphi_{1}|\nabla v|^{2}+s^{3} \lambda^{4} \varphi_{1}^{3} v^{2}\right\} \mathrm{e}^{2 s \varphi_{1}} \mathrm{~d} x \mathrm{~d} t \\
\leq & \int_{Q} F^{2} \mathrm{e}^{2 s \varphi_{1}} \mathrm{~d} x \mathrm{~d} t+C \int_{D_{1}} \sum_{j=1}^{\ell} \chi^{2}(x, t)\left|\partial_{t}^{\alpha_{j}} u\right|^{2} \mathrm{e}^{2 s \varphi_{1}} \mathrm{~d} x \mathrm{~d} t+C \int_{D_{1}}\left|A_{1} u\right|^{2} \mathrm{e}^{2 s \varphi_{1}} \mathrm{~d} x \mathrm{~d} t \\
& +\mathrm{e}^{C(\lambda) s} \int_{\Sigma_{0} \cup(\Gamma \times(0, T))}\left(|\nabla v|^{2}+v^{2}\right) \mathrm{d} \Sigma+\mathrm{e}^{C(\lambda) s} \int_{\Gamma \times(0, T)}\left|\partial_{t} v\right|^{2} \mathrm{~d} S \mathrm{~d} t .
\end{aligned}
$$

for all $s \geq s_{0}$ and $\lambda \geq \lambda_{0}$.

By (23), $A_{1} u$ does not vanish only if $\mu_{2} \leq \varphi(x, t) \leq \mu_{3}$ and so

$$
\int_{D_{1}}\left|A_{1} u\right|^{2} \mathrm{e}^{2 s \varphi_{1}} \mathrm{~d} x \mathrm{~d} t \leq C \mathrm{e}^{2 s \mu_{3}}\|u\|_{H^{1,0}(Q)}^{2} .
$$

Moreover, from (21) and Lemma 2.1. letting $\mathcal{C}_{1}=\left\{(x, t) ; x \in \bar{\Omega}, t>0, \varphi_{1}(x, t)>\frac{\mu_{3}+\mu_{4}}{2}\right\}$ and $\mathcal{C}_{2}=D_{3}$ in Lemma 2.1, for $\lambda$ being large fixed, we conclude that the integration $\int_{\mathcal{C}_{1}} \sum_{j=1}^{\ell} \chi^{2}(x, t)\left|\partial_{t}^{\alpha_{j}} u\right|^{2} \mathrm{e}^{2 s \varphi_{1}} \mathrm{~d} x \mathrm{~d} t$ can be absorbed by the left-hand side of (25), which implies

$$
\begin{aligned}
& \int_{D_{3}}\left\{\frac{1}{s \varphi_{1}}\left|\partial_{t} u\right|^{2}+s \varphi_{1}|\nabla u|^{2}+s^{3} \varphi_{1}^{3} u^{2}\right\} \mathrm{e}^{2 s \varphi_{1}} \mathrm{~d} x \mathrm{~d} t \\
\leq & C \mathrm{e}^{C s}\|F\|_{L^{2}(Q)}+C \mathrm{e}^{2 s \mu_{3}}\|u\|_{H^{1,0}(Q)}^{2}+C \sum_{j=1}^{\ell} \int_{D_{1} \backslash \mathcal{C}_{1}}\left|\partial_{t}^{\alpha_{j}} u\right|^{2} \mathrm{e}^{2 s \varphi_{1}} \mathrm{~d} x \mathrm{~d} t \\
& +\mathrm{e}^{C s} \int_{\Sigma_{0} \cup(\Gamma \times(0, T))}\left(|\nabla v|^{2}+v^{2}\right) \mathrm{d} \Sigma+\mathrm{e}^{C s} \int_{\Gamma \times(0, T)}\left|\partial_{t} v\right|^{2} \mathrm{~d} S \mathrm{~d} t .
\end{aligned}
$$

By (20), we can directly verify that $\varphi_{1}(x, t) \leq \frac{\mu_{3}+\mu_{4}}{2}$ in $D_{1} \backslash \mathcal{C}_{1}$, and if $(x, t) \in \Omega_{0} \times(0, \varepsilon)$, then $\varphi_{1}(x, t)>\mu_{4}$. Then combined with (21) and (22), by Hölder's inequality, we have

$$
\begin{aligned}
& \mathrm{e}^{2 s \mu_{4}} \int_{0}^{\frac{T}{2 M}} \int_{\Omega_{0}}\left\{\frac{1}{s}\left|\partial_{t} u\right|^{2}+s|\nabla u|^{2}+s^{3} u^{2}\right\} \mathrm{d} x \mathrm{~d} t \\
\leq & C \mathrm{e}^{C s}\|F\|_{L^{2}(Q)}+C \mathrm{e}^{2 s \mu_{3}}\|u\|_{H^{1,0}(Q)}^{2}+C \mathrm{e}^{2 s \frac{\mu_{3}+\mu_{4}}{2}} \int_{Q}\left|\partial_{t} u\right|^{2} \mathrm{~d} x \mathrm{~d} t
\end{aligned}
$$




$$
+\mathrm{e}^{C s} \int_{\Omega}\left(|\nabla v(x, 0)|^{2}+v^{2}(x, 0)\right) \mathrm{d} x+\mathrm{e}^{C s} \int_{\Gamma \times(0, T)}\left(\left|\partial_{t} v\right|^{2}+|\nabla v|^{2}+v^{2}\right) \mathrm{d} S \mathrm{~d} t .
$$

for $s \geq s_{0}$. Then dividing both sides by $\mathrm{e}^{2 s \mu_{4}}$, since

$$
s \mathrm{e}^{-2 s \frac{\mu_{4}-\mu_{3}}{2}} \leq C \mathrm{e}^{-\frac{s\left(\mu_{4}-\mu_{3}\right)}{2}}, \quad s \mathrm{e}^{-2 s\left(\mu_{4}-\mu_{3}\right)} \leq C \mathrm{e}^{-s\left(\mu_{4}-\mu_{3}\right)} \leq C \mathrm{e}^{-\frac{s\left(\mu_{4}-\mu_{3}\right)}{2}},
$$

by replacing $C$ by $C \mathrm{e}^{C s_{0}}$, we have

$$
\|u\|_{H^{1,1}\left(\Omega_{0} \times\left(0, \frac{T}{2 M}\right)\right)}^{2} \leq C \mathrm{e}^{-\frac{\mu_{4}-\mu_{3}}{2} s}\|u\|_{H^{1,1}(Q)}^{2}+C \mathrm{e}^{C s} \mathcal{D}^{2}
$$

for all $s \geq 0$ and $u \in H^{2,1}(Q)$, where the constant $C>0$ depends on $T, \Omega_{0}$ and the coefficients of the equation (2).

Firstly, if $\mathcal{D}=0$, letting $s \rightarrow \infty$, we conclude that $u=0$ in $\Omega_{0} \times\left(0, \frac{T}{2 M}\right)$, so that the conclusion of Theorem 2 holds true. Next let $\mathcal{D} \neq 0$. First let $\mathcal{D} \geq\|u\|_{H^{1,1}(Q)}$. Then (26) implies

$$
\|u\|_{H^{1,1}\left(\Omega_{0} \times\left(0, \frac{T}{2 M}\right)\right)} \leq C \mathrm{e}^{C s} \mathcal{D}, \quad s \geq 0,
$$

which already proves the theorem. Second let $\mathcal{D} \leq\|u\|_{H^{1,1}(Q)}$, we choose $s>0$ minimizing the right-hand side of (26), that is

$$
\mathrm{e}^{-\frac{\mu_{4}-\mu_{3}}{2} s}\|u\|_{H^{1,1}(Q)}^{2}=\mathrm{e}^{C s} \mathcal{D}^{2} .
$$

By $\mathcal{D} \neq 0$, we can choose

$$
s=\frac{4}{2 C+\mu_{4}-\mu_{3}} \log \frac{\|u\|_{H^{1,1}(Q)}}{\mathcal{D}}>0 .
$$

Then (26) gives

$$
\|u\|_{H^{1,1}\left(\Omega_{0} \times\left(0, \frac{T}{2 M}\right)\right)} \leq 2 C\|u\|_{H^{1,1}(Q)}^{1-\theta} \mathcal{D}^{\theta},
$$

where $\theta:=\frac{\mu_{4}-\mu_{3}}{2 C+\mu_{4}-\mu_{3}}$, and the constant $C$ depends on $\Omega_{0}, T$ and the coefficients of the equation (2). We complete the proof of the theorem by setting $\varepsilon=\frac{T}{2 M}$.

\section{Carleman estimate for a sup-diffusion and its applications}

In this section, we pay attention to the Carleman estimate for the equation (7) in the case of $\alpha=\frac{m}{k}$, say,

$$
\partial_{t} u+q(x) \partial_{t}^{\frac{m}{k}} u-\Delta u+B \cdot \nabla u+c u=F \quad \text { in } Q
$$

and its applications to the lateral Cauchy problem and the inverse source problem. Without loss of generality, we set $a_{i j}=\delta_{i j}, 1 \leq i, j \leq n$ here. In the following two subsections, we will give the proofs of Theorems 3 , 廿4 and 5 respectively.

\subsection{Carleman estimate for a sup-diffusion}

In this subsection, we will give a proof of Theorem 3 Similar to the case of $\alpha<\frac{1}{2}$, we construct the Carleman estimate for the following parabolic type equation

$$
\partial_{t} u-\Delta u+B \cdot \nabla u+c u=F-q(x) \partial_{t}^{\alpha} u,
$$

whereas here we further multiply on both sides of the above equation by several Riemann-Liouville fractional derivatives in order to dealing the new source term $\partial_{t}^{\alpha} u$. We have the following details of the proof. 
Proof of Theorem 3. For some technical reasons, we divide the proof into two cases:

(i) $\alpha=\frac{m}{2 k+1}, m, k \in \mathbb{Z}, k \geq 1, m=1,2, \ldots, 2 k$,

(ii) $\alpha=\frac{m}{2 k}, m, k \in \mathbb{Z}, k \geq 1, m=1,2, \ldots, 2 k-1$.

In the first part, we consider the case (i) in which the denominator is odd. Because of the zero initial condition, the equation (28) can be rephrased as

$$
\widetilde{L} u:=\partial_{t} u-\Delta u+B \cdot \nabla u+c u=F-q(x) D_{t}^{\frac{m}{2 k+1}} u \quad \text { in } Q .
$$

Recalling the definition of Riemann-Liouville fractional integral operator $D_{t}^{-p}$ :

$$
D_{t}^{-p} u:={ }_{0} D_{t}^{-p} u=\frac{1}{\Gamma(p)} \int_{0}^{t}(t-s)^{p-1} u(s) \mathrm{d} s,
$$

and we have the semigroup property for the R.- L. fractional integral operators:

$$
D_{t}^{-p_{1}} D_{t}^{-p_{2}} u=D_{t}^{-p_{1}-p_{2}} u, \quad \forall p_{1}, p_{2}>0 .
$$

We first apply the fractional differential(or integral) operators $D_{t}^{\frac{j}{2 k+1}}$ to the equation (29) separately for $j=-k, \ldots, k$ to derive

$$
D_{t}^{\frac{j}{2 k+1}}\left(\partial_{t} u\right)-D_{t}^{\frac{j}{2 k+1}}(\Delta u)+D_{t}^{\frac{j}{2 k+1}}(B \cdot \nabla u)+D_{t}^{\frac{j}{2 k+1}}(c u)=D_{t}^{\frac{j}{2 k+1}} F-D_{t}^{\frac{j}{2 k+1}}\left(q D_{t}^{\frac{m}{2 k+1}} u\right), \quad j=-k, \ldots, k .
$$

Moreover, the homogeneous initial value implies that the differential operators and the R.-L. integral operator are commutable, which along with the formula $D_{t}^{\frac{j}{2 k+1}}\left(D_{t}^{\frac{m}{2 k+1}} u\right)=D_{t}^{\frac{j}{2 k+1}+\frac{m}{2 k+1}} u=D_{t}^{\frac{j+m}{2 k+1}} u$ implies that

$$
\widetilde{L}\left(u_{j}\right)=\partial_{t} u_{j}-\Delta u_{j}+B \cdot \nabla u_{j}+c u_{j}=D_{t}^{\frac{j}{2 k+1}} F-q D_{t}^{\frac{j+m}{2 k+1}} u, \quad j=-k, \ldots, k
$$

where $u_{j}:=D_{t}^{\frac{j}{2 k+1}} u$, for all $j \in \mathbb{Z}$. Next we denote $\widetilde{u_{j}}:=\chi_{0} u_{j}$. Then by noting that

$$
\widetilde{L}\left(\chi_{0} u\right)-\chi_{0} \widetilde{L} u=\left(\partial_{t} \chi_{0}\right) u-2 \nabla \chi_{0} \cdot \nabla u-\left(\Delta \chi_{0}\right) u+\left(B \cdot \nabla \chi_{0}\right) u
$$

the equations (30) can be rewritten by:

$$
\widetilde{L}\left(\widetilde{u_{j}}\right)=\chi_{0} D_{t}^{\frac{j}{2 k+1}} F-\left(\chi_{0} q\right) u_{j+m}-2 \nabla \chi_{0} \cdot \nabla u_{j}+\left(B \cdot \nabla \chi_{0}-\Delta \chi_{0}+\partial_{t} \chi_{0}\right) u_{j}
$$

for all $j=-k, \ldots, k$. Now we use a Carleman estimate for parabolic type stated in the following lemma.

Lemma 3.1. Let $F \in L^{2}(Q)$. Then there exist constants $\hat{\lambda} \geq 1, \hat{s} \geq 1$ and $C>0, C(\lambda)>0$ such that

$$
\begin{gathered}
\int_{Q}\left\{s^{\tau-1} \lambda^{\tau} \varphi_{2}^{\tau-1}\left(\left|\partial_{t} u\right|^{2}+\sum_{i, j=1}^{n}\left|\partial_{i} \partial_{j} u\right|^{2}\right)+s^{\tau+1} \lambda^{\tau+2} \varphi_{2}^{\tau+1}|\nabla u|^{2}+s^{\tau+3} \lambda^{\tau+4} \varphi_{2}^{\tau+3}|u|^{2}\right\} e^{2 s \varphi_{2}} \mathrm{~d} x \mathrm{~d} t \\
\leq C \int_{Q}\left(s \lambda \varphi_{2}\right)^{\tau}|F|^{2} e^{2 s \varphi_{2}} \mathrm{~d} x \mathrm{~d} t+C(\lambda) e^{C(\lambda) s} s^{\tau} \int_{\partial Q}\left(\left|\nabla_{x, t} u\right|^{2}+|u|^{2}\right) \mathrm{d} S \mathrm{~d} t
\end{gathered}
$$

for all $s \geq \hat{s}, \lambda \geq \hat{\lambda}, \tau \in \mathbb{Z}$ and all $u$ smooth enough satisfying the equation:

$$
\widetilde{L} u=F \text {. }
$$

The proof of this lemma is similar to that of Theorem 3.2 in [18. However we need to calculate more carefully when we do the integration by parts because we have a new weight $\varphi_{2}^{\tau}$. To specify the dependence, $C$ and $\hat{\lambda}$ depends on $\|\nabla d\|$ while $\hat{s}$ also depends on $\tau, \beta, T$ and the coefficients of the operator $\widetilde{L}$. Furthermore, $C(\lambda)$ even depends on large parameter $\lambda$ but is independent of $s$. 
Applying the above lemma to the equations (31), we have the following Carleman estimates:

$$
\begin{aligned}
& \lambda \int_{Q}\left\{\left(s \lambda \varphi_{2}\right)^{\tau_{j}-1}\left(\left|\partial_{t} \widetilde{u_{j}}\right|^{2}+\sum_{i, l=1}^{n}\left|\partial_{i} \partial_{l} \widetilde{u_{j}}\right|^{2}\right)+\left(s \lambda \varphi_{2}\right)^{\tau_{j}+1}\left|\nabla \widetilde{u_{j}}\right|^{2}+\left(s \lambda \varphi_{2}\right)^{\tau_{j}+3}\left|\widetilde{u_{j}}\right|^{2}\right\} e^{2 s \varphi_{2}} \mathrm{~d} x \mathrm{~d} t \\
& \leq C \int_{Q}\left(s \lambda \varphi_{2}\right)^{\tau_{j}}\left(\left|\chi_{0} D_{t}^{\frac{j}{2 k+1}} F\right|^{2}+\left|\chi_{0} u_{j+m}\right|^{2}\right) e^{2 s \varphi_{2}} \mathrm{~d} x \mathrm{~d} t+C \int_{Q}\left(s \lambda \varphi_{2}\right)^{\tau_{j}}\left(\left|\widetilde{L}\left(\chi_{0} u_{j}\right)-\chi_{0} \widetilde{L}\left(u_{j}\right)\right|^{2}\right) e^{2 s \varphi_{2}} \mathrm{~d} x \mathrm{~d} t \\
& \quad+C(\lambda) s^{\tau_{j}} e^{C(\lambda) s} \int_{\partial Q}\left(\left|\nabla_{x, t}\left(\chi_{0} u_{j}\right)\right|^{2}+\left|\chi_{0} u_{j}\right|^{2}\right) \mathrm{d} S \mathrm{~d} t
\end{aligned}
$$

for all $\lambda \geq \lambda_{j}, s \geq s_{j}$ and $j=-k, . ., k$.

Moreover, we observe that a direct calculation implies that

$$
\begin{aligned}
\left|\partial_{t} \widetilde{u_{j}}\right|^{2} & =\left|\left(\partial_{t} \chi_{0}\right) u_{j}+\chi_{0}\left(\partial_{t} u_{j}\right)\right|^{2} \geq \frac{1}{2}\left|\chi_{0}\left(\partial_{t} u_{j}\right)\right|^{2}-\left|\left(\partial_{t} \chi_{0}\right) u_{j}\right|^{2}, \\
\left|\partial_{i} \partial_{l} \widetilde{u_{j}}\right|^{2} & =\left|\chi_{0} \partial_{i} \partial_{l} u_{j}+\left(\partial_{i} \chi_{0}\right)\left(\partial_{l} u_{j}\right)+\left(\partial_{l} \chi_{0}\right)\left(\partial_{i} u_{j}\right)+\left(\partial_{i} \partial_{l} \chi_{0}\right) u_{j}\right|^{2} \\
& \geq \frac{1}{2}\left|\chi_{0} \partial_{i} \partial_{l} u_{j}\right|^{2}-3\left|\left(\partial_{i} \chi_{0}\right)\left(\partial_{l} u_{j}\right)\right|^{2}-3\left|\left(\partial_{i} \chi_{0}\right)\left(\partial_{l} u_{j}\right)\right|^{2}-3\left|\left(\partial_{i} \partial_{l} \chi_{0}\right) u_{j}\right|^{2}, \\
\left|\nabla \widetilde{u_{j}}\right|^{2} & =\left|\chi_{0} \nabla u_{j}+u_{j} \nabla \chi_{0}\right|^{2} \geq \frac{1}{2}\left|\chi_{0} \nabla u_{j}\right|^{2}-\left|\nabla \chi_{0}\right|^{2}\left|u_{j}\right|^{2} .
\end{aligned}
$$

Thus, Carleman inequalities (32) lead to

$\lambda \int_{Q} \chi_{0}^{2}\left\{\left(s \lambda \varphi_{2}\right)^{\tau_{0}-1}\left(\left|\partial_{t} u\right|^{2}+\sum_{i, l=1}^{n}\left|\partial_{i} \partial_{l} u\right|^{2}\right)+\left(s \lambda \varphi_{2}\right)^{\tau_{0}+1}|\nabla u|^{2}+\left(s \lambda \varphi_{2}\right)^{\tau_{0}+3}|u|^{2}\right\} e^{2 s \varphi_{2}} \mathrm{~d} x \mathrm{~d} t$
$\leq C \int_{Q} \chi_{0}^{2}\left(s \lambda \varphi_{2}\right)^{\tau_{0}}\left(|F|^{2}+\left|u_{m}\right|^{2}\right) e^{2 s \varphi_{2}} \mathrm{~d} x \mathrm{~d} t+C(\lambda) s^{\tau_{0}} e^{C(\lambda) s} \int_{\partial Q}\left(\left|\nabla_{x, t}\left(\chi_{0} u\right)\right|^{2}+\left|\chi_{0} u\right|^{2}\right) \mathrm{d} S \mathrm{~d} t$
$+C \int_{Q}\left(s \lambda \varphi_{2}\right)^{\tau_{0}}\left(\left|\widetilde{L}\left(\chi_{0} u\right)-\chi_{0} \widetilde{L} u\right|^{2}+\left(\left|\partial_{t} \chi_{0}\right|^{2}+s \lambda^{2} \varphi_{2}\left|\nabla \chi_{0}\right|^{2}+\sum_{i, l=1}^{n}\left|\partial_{i} \partial_{l} \chi_{0}\right|^{2}\right)|u|^{2}+\left|\nabla \chi_{0}\right|^{2}|\nabla u|^{2}\right) e^{2 s \varphi_{2}} \mathrm{~d} x \mathrm{~d} t$ for all $\lambda \geq \lambda_{0}, s \geq s_{0}$ and

$$
\begin{aligned}
& \lambda \int_{Q} \chi_{0}^{2}\left\{\left(s \lambda \varphi_{2}\right)^{\tau_{j}-1}\left|\partial_{t} u_{j}\right|^{2}+\left(s \lambda \varphi_{2}\right)^{\tau_{j}+1}\left|\nabla u_{j}\right|^{2}+\left(s \lambda \varphi_{2}\right)^{\tau_{j}+3}\left|u_{j}\right|^{2}\right\} e^{2 s \varphi_{2}} \mathrm{~d} x \mathrm{~d} t \\
& \leq C \int_{Q} \chi_{0}^{2}\left(s \lambda \varphi_{2}\right)^{\tau_{j}}\left(\left|D_{t}^{\frac{j}{2 k+1}} F\right|^{2}+\left|u_{j+m}\right|^{2}\right) e^{2 s \varphi_{2}} \mathrm{~d} x \mathrm{~d} t+C(\lambda) s^{\tau_{j}} e^{C(\lambda) s} \int_{\partial Q}\left(\left|\nabla_{x, t}\left(\chi_{0} u_{j}\right)\right|^{2}+\left|\chi_{0} u_{j}\right|^{2}\right) \mathrm{d} S \mathrm{~d} t \\
& +C \int_{Q}\left(s \lambda \varphi_{2}\right)^{\tau_{j}}\left(\left|\widetilde{L}\left(\chi_{0} u_{j}\right)-\chi_{0} \widetilde{L}\left(u_{j}\right)\right|^{2}+\left|\partial_{t} \chi_{0}\right|^{2}\left|u_{j}\right|^{2}+s \lambda^{2} \varphi_{2}\left|\nabla \chi_{0}\right|^{2}\left|u_{j}\right|^{2}\right) e^{2 s \varphi_{2}} \mathrm{~d} x \mathrm{~d} t
\end{aligned}
$$

for all $\lambda \geq \lambda_{j}, s \geq s_{j}$ and all $j=-k, \ldots,-1,1, \ldots, k$. Combining the Carleman inequalities (33)-(34), i.e., taking the summation, we obtain

$$
\begin{aligned}
& \lambda \int_{Q} \chi_{0}^{2}\left\{\left(s \lambda \varphi_{2}\right)^{\tau_{0}-1} \sum_{i, l=1}^{n}\left|\partial_{i} \partial_{l} u\right|^{2}+\left(s \lambda \varphi_{2}\right)^{\tau_{0}+1}|\nabla u|^{2}+\sum_{j=-k}^{k}\left(\left(s \lambda \varphi_{2}\right)^{\tau_{j}-1}\left|\partial_{t} u_{j}\right|^{2}+\left(s \lambda \varphi_{2}\right)^{\tau_{j}+3}\left|u_{j}\right|^{2}\right)\right\} e^{2 s \varphi_{2}} \mathrm{~d} x \mathrm{~d} t \\
& \leq C \int_{Q} \chi_{0}^{2} \sum_{j=-k}^{k}\left(s \lambda \varphi_{2}\right)^{\tau_{j}}\left|D_{t}^{\frac{j}{2 k+1}} F\right|^{2} e^{2 s \varphi_{2}} \mathrm{~d} x \mathrm{~d} t+C \int_{Q} \chi_{0}^{2} \sum_{j=-k}^{k}\left(s \lambda \varphi_{2}\right)^{\tau_{j}}\left|u_{j+m}\right|^{2} e^{2 s \varphi_{2}} \mathrm{~d} x \mathrm{~d} t+\text { Low1 }+ \text { Bdy } 1
\end{aligned}
$$

for all $\lambda \geq \tilde{\lambda}:=\max \left\{\lambda_{j}:-k \leq j \leq k\right\}$ and $s \geq \tilde{s}:=\max \left\{s_{j}:-k \leq j \leq k\right\}$. Here Low1 and Bdy1 are the lower order terms and boundary terms determined as

$$
L o w 1=C \int_{Q} \sum_{j=-k}^{k}\left(s \lambda \varphi_{2}\right)^{\tau_{j}}\left(\left|\partial_{t} \chi_{0}\right|^{2}+\left|\nabla \chi_{0}\right|^{2}+\sum_{i, l=1}^{n}\left|\partial_{i} \partial_{l} \chi_{0}\right|^{2}\right)\left(\left|\nabla u_{j}\right|^{2}+s \lambda^{2} \varphi_{2}\left|u_{j}\right|^{2}\right) \mathrm{e}^{2 s \varphi_{2}} \mathrm{~d} x \mathrm{~d} t,
$$




$$
B d y 1=C(\lambda) \mathrm{e}^{C(\lambda) s} \int_{\partial Q} \sum_{j=-k}^{k} s^{\tau_{j}}\left(\left|\partial_{t} \chi_{0}\right|^{2}+\left|\nabla \chi_{0}\right|^{2}+\left|\chi_{0}\right|^{2}\right)\left(\left|\nabla_{x, t} u_{j}\right|^{2}+\left|u_{j}\right|^{2}\right) \mathrm{d} S \mathrm{~d} t .
$$

Now we fix $\tau_{j}=-\frac{4}{2 k+1}(j+k) \leq 0, j=-k, \ldots, k$. Along with the relation

$$
u_{j+2 k+1}=D_{t}^{\frac{j+2 k+1}{2 k+1}} u=\partial_{t}\left(D_{t}^{\frac{j}{2 k+1}} u\right)=\partial_{t} u_{j}
$$

which follows after the zero initial condition, a direct calculation yields that

$$
\begin{aligned}
& \sum_{j=-k}^{k}\left(s \lambda \varphi_{2}\right)^{\tau_{j}-1}\left|u_{j+2 k+1}\right|^{2}=\sum_{j=-k}^{k}\left(s \lambda \varphi_{2}\right)^{-\frac{4 j+6 k+1}{2 k+1}}\left|u_{j+2 k+1}\right|^{2}=\sum_{j=k+1}^{3 k+1}\left(s \lambda \varphi_{2}\right)^{-\frac{4 j-2 k-3}{2 k+1}}\left|u_{j}\right|^{2}, \\
& \sum_{j=-k}^{k}\left(s \lambda \varphi_{2}\right)^{\tau_{j}+3}\left|u_{j}\right|^{2}=\sum_{j=-k}^{k}\left(s \lambda \varphi_{2}\right)^{-\frac{4 j-2 k-3}{2 k+1}}\left|u_{j}\right|^{2}, \\
& \sum_{j=-k}^{k}\left(s \lambda \varphi_{2}\right)^{\tau_{j}}\left|u_{j+m}\right|^{2}=\sum_{j=-k}^{k}\left(s \lambda \varphi_{2}\right)^{-\frac{4 j+4 k}{2 k+1}}\left|u_{j+m}\right|^{2}=\sum_{j=-k+m}^{k+m}\left(s \lambda \varphi_{2}\right)^{-\frac{4 j+4 k-4 m}{2 k+1}}\left|u_{j}\right|^{2},
\end{aligned}
$$

which imply that

$$
\begin{aligned}
& \lambda \int_{Q} \chi_{0}^{2}\left\{\sum_{i, l=1}^{n}\left(s \lambda \varphi_{2}\right)^{-\frac{6 k+1}{2 k+1}}\left|\partial_{i} \partial_{l} u\right|^{2}+\left(s \lambda \varphi_{2}\right)^{-\frac{2 k-1}{2 k+1}}|\nabla u|^{2}+\sum_{j=-k}^{3 k+1}\left(s \lambda \varphi_{2}\right)^{-\frac{4 j-2 k-3}{2 k+1}}\left|u_{j}\right|^{2}\right\} e^{2 s \varphi_{2}} \mathrm{~d} x \mathrm{~d} t \\
& \leq C \int_{Q} \chi_{0}^{2} \sum_{j=-k}^{k}\left(s \lambda \varphi_{2}\right)^{-\frac{4 j+4 k}{2 k+1}}\left|D_{t}^{\frac{j}{2 k+1}} F\right|^{2} e^{2 s \varphi_{2}} \mathrm{~d} x \mathrm{~d} t+C \int_{Q} \chi_{0}^{2} \sum_{j=-k+m}^{k+m}\left(s \lambda \varphi_{2}\right)^{-\frac{4 j+4 k-4 m}{2 k+1}}\left|u_{j}\right|^{2} e^{2 s \varphi_{2}} \mathrm{~d} x \mathrm{~d} t \\
& \quad+\text { Low } 1+B d y 1
\end{aligned}
$$

for all $\lambda \geq \widetilde{\lambda}$ and all $s \geq \widetilde{s}$. Since $\alpha=\frac{m}{2 k+1} \in\left(0, \frac{3}{4}\right]$ implies

$$
-\frac{4 j-2 k-3}{2 k+1} \geq-\frac{4 j+4 k-4 m}{2 k+1} \text { for } j=-k+m, \ldots, k+m,
$$

we then fix $\lambda$ large so that the second terms on the RHS of (35) can be absorbed into the LHS of it $(\lambda \geq \hat{\lambda})$. Moreover, we have

$$
\begin{aligned}
& \text { Low } 1 \leq C \int_{Q} \sum_{j=-k}^{k}\left(\left|\partial_{t} \chi_{0}\right|^{2}+\left|\nabla \chi_{0}\right|^{2}+\sum_{i, l=1}^{n}\left|\partial_{i} \partial_{l} \chi_{0}\right|^{2}\right)\left(\left|\nabla u_{j}\right|^{2}+s \varphi_{2}\left|u_{j}\right|^{2}\right) \mathrm{e}^{2 s \varphi_{2}} \mathrm{~d} x \mathrm{~d} t \leq \text { Low }, \\
& B d y 1 \leq C \mathrm{e}^{C s} \int_{\partial Q} \sum_{j=-k}^{k}\left(\left|\partial_{t} \chi_{0}\right|^{2}+\left|\nabla \chi_{0}\right|^{2}+\left|\chi_{0}\right|^{2}\right)\left(\left|\nabla_{x, t} u_{j}\right|^{2}+\left|u_{j}\right|^{2}\right) \mathrm{d} S \mathrm{~d} t=B d y
\end{aligned}
$$

since $s, \lambda, \varphi_{2} \geq 1$ and $\tau_{j} \leq 0$. Therefore, (35) yields

$$
\begin{aligned}
& \int_{Q} \chi_{0}^{2}\left\{\sum_{i, l=1}^{n}\left(s \varphi_{2}\right)^{-\frac{6 k+1}{2 k+1}}\left|\partial_{i} \partial_{l} u\right|^{2}+\left(s \varphi_{2}\right)^{-\frac{2 k-1}{2 k+1}}|\nabla u|^{2}+\sum_{j=-k}^{3 k+1}\left(s \varphi_{2}\right)^{-\frac{4 j-2 k-3}{2 k+1}}\left|D_{t}^{\frac{j}{2 k+1}} u\right|^{2}\right\} e^{2 s \varphi_{2}} \mathrm{~d} x \mathrm{~d} t \\
& \leq C \int_{Q} \chi_{0}^{2} \sum_{j=-k}^{k}\left(s \varphi_{2}\right)^{-\frac{4 j+4 k}{2 k+1}}\left|D_{t}^{\frac{j}{2 k+1}} F\right|^{2} e^{2 s \varphi_{2}} \mathrm{~d} x \mathrm{~d} t+L o w+B d y
\end{aligned}
$$

for $\lambda$ large fixed and all $s \geq \widetilde{s}$.

For the case (ii), we repeat the strategy of above to obtain the equations similar to (31):

$$
\widetilde{L}\left(\widetilde{u_{j}}\right)=\chi_{0} D_{t}^{\frac{j}{2 k}} F-\left(\chi_{0} q\right) u_{j+m}-2 \nabla \chi_{0} \cdot \nabla u_{j}+\left(B \cdot \nabla \chi_{0}-\Delta \chi_{0}+\partial_{t} \chi_{0}\right) u_{j}
$$


for all $j=-k+1, \ldots, k$ where we denote $u_{j}:=D_{t}^{\frac{j}{2 k}} u$ and $\widetilde{u_{j}}=\chi_{0} u_{j}$ for all $j \in \mathbb{Z}$ instead. Again we apply Carleman estimate for parabolic equations (Lemma 3.1) to (36) and take the summation over $j$ from $-k+1$ to $k$ which reads

$$
\begin{aligned}
& \lambda \int_{Q} \chi_{0}^{2}\left\{\left(s \lambda \varphi_{2}\right)^{\tau_{0}-1} \sum_{i, l=1}^{n}\left|\partial_{i} \partial_{l} u\right|^{2}+\left(s \lambda \varphi_{2}\right)^{\tau_{0}+1}|\nabla u|^{2}+\sum_{j=-k+1}^{k}\left(\left(s \lambda \varphi_{2}\right)^{\tau_{j}-1}\left|u_{j+2 k}\right|^{2}+\left(s \lambda \varphi_{2}\right)^{\tau_{j}+3}\left|u_{j}\right|^{2}\right)\right\} e^{2 s \varphi_{2}} \mathrm{~d} x \mathrm{~d} t \\
& \leq C \int_{Q} \chi_{0}^{2} \sum_{j=-k+1}^{k}\left(s \lambda \varphi_{2}\right)^{\tau_{j}}\left|D_{t}^{\frac{j}{2 k}} F\right|^{2} e^{2 s \varphi_{2}} \mathrm{~d} x \mathrm{~d} t+C \int_{Q} \chi_{0}^{2} \sum_{j=-k+1}^{k}\left(s \lambda \varphi_{2}\right)^{\tau_{j}}\left|u_{j+m}\right|^{2} e^{2 s \varphi_{2}} \mathrm{~d} x \mathrm{~d} t+\text { Low } 2+\text { Bdy } 2
\end{aligned}
$$

for all $\lambda \geq \widetilde{\lambda}$ and $s \geq \widetilde{s}$. Here Low2 and Bdy2 are the lower order terms and boundary terms determined as

$$
\begin{aligned}
& \text { Low } 2=C \int_{Q_{j}} \sum_{j=-k+1}^{k}\left(s \lambda \varphi_{2}\right)^{\tau_{j}}\left(\left|\partial_{t} \chi_{0}\right|^{2}+\left|\nabla \chi_{0}\right|^{2}+\sum_{i, l=1}^{n}\left|\partial_{i} \partial_{l} \chi_{0}\right|^{2}\right)\left(\left|\nabla u_{j}\right|^{2}+s \lambda^{2} \varphi_{2}\left|u_{j}\right|^{2}\right) \mathrm{e}^{2 s \varphi_{2}} \mathrm{~d} x \mathrm{~d} t, \\
& B d y 2=C(\lambda) \mathrm{e}^{C(\lambda) s} \int_{\partial Q_{j=-k+1}} \sum_{j}^{k} s^{\tau_{j}}\left(\left|\partial_{t} \chi_{0}\right|^{2}+\left|\nabla \chi_{0}\right|^{2}+\left|\chi_{0}\right|^{2}\right)\left(\left|\nabla_{x, t} u_{j}\right|^{2}+\left|u_{j}\right|^{2}\right) \mathrm{d} S \mathrm{~d} t .
\end{aligned}
$$

For the case (ii), we choose $\tau_{j}=-\frac{2}{k}(j+k-1) \leq 0, j=-k+1, \ldots, k$. By direct calculation, we obtain

$$
\begin{aligned}
& \sum_{j=-k+1}^{k}\left(s \lambda \varphi_{2}\right)^{\tau_{j}-1}\left|u_{j+2 k}\right|^{2}=\sum_{j=-k+1}^{k}\left(s \lambda \varphi_{2}\right)^{-\frac{2 j+3 k-2}{k}}\left|u_{j+2 k}\right|^{2}=\sum_{j=k+1}^{3 k}\left(s \lambda \varphi_{2}\right)^{-\frac{2 j-k-2}{k}}\left|u_{j}\right|^{2}, \\
& \sum_{j=-k+1}^{k}\left(s \lambda \varphi_{2}\right)^{\tau_{j}+3}\left|u_{j}\right|^{2}=\sum_{j=-k+1}^{k}\left(s \lambda \varphi_{2}\right)^{-\frac{2 j-k-2}{k}\left|u_{j}\right|^{2},} \\
& \sum_{j=-k+1}^{k}\left(s \lambda \varphi_{2}\right)^{\tau_{j}}\left|u_{j+m}\right|^{2}=\sum_{j=-k+1}^{k}\left(s \lambda \varphi_{2}\right)^{-\frac{2 j+2 k-2}{k}}\left|u_{j+m}\right|^{2}=\sum_{j=-k+m+1}^{k+m}\left(s \lambda \varphi_{2}\right)^{-\frac{2 j+2 k-2 m-2}{k}}\left|u_{j}\right|^{2} .
\end{aligned}
$$

Since $\alpha=\frac{m}{2 k} \in\left(0, \frac{3}{4}\right]$ implies that

$$
-\frac{2 j-k-2}{k} \geq-\frac{2 j+2 k-2 m-2}{k} \text { for } j=-k+m+1, \ldots, k+m,
$$

we fix $\lambda$ so large that the second terms on the RHS of (38) can be absorbed. Therefore, we have the following Carleman inequality

$$
\begin{aligned}
& \int_{Q} \chi_{0}^{2}\left\{\sum_{i, l=1}^{n}\left(s \varphi_{2}\right)^{-\frac{3 k-2}{k}}\left|\partial_{i} \partial_{l} u\right|^{2}+\left(s \varphi_{2}\right)^{-\frac{k-2}{k}}|\nabla u|^{2}+\sum_{j=-k}^{3 k}\left(s \varphi_{2}\right)^{-\frac{2 j-k-2}{k}}\left|D_{t}^{\frac{j}{2 k}} u\right|^{2}\right\} e^{2 s \varphi_{2}} \mathrm{~d} x \mathrm{~d} t \\
& \leq C \int_{Q} \chi_{0}^{2} \sum_{j=-k+1}^{k}\left(s \varphi_{2}\right)^{-\frac{2 j+2 k-2}{k}}\left|D_{t}^{\frac{j}{2 k}} F\right|^{2} e^{2 s \varphi_{2}} \mathrm{~d} x \mathrm{~d} t+\text { Low }+ \text { Bdy }
\end{aligned}
$$

for $\lambda$ large fixed and all $s \geq \widetilde{s}$.

Theorem 3 now follows after the two Carleman inequlaities (36) and (39).

Remark 3.1. Under the assumption $h(0)=0$, we note that

$$
\left\|D_{t}^{\alpha} h\right\|_{L^{2}(0, T)} \leq C\left\|D_{t}^{\beta} h\right\|_{L^{2}(0, T)}, \quad-1<\alpha<\beta<2,
$$

which actually can be derived by using the definition of $D_{t}^{\alpha}$ and Young's inequality. In view of this estimate, we can also rewrite Bdy as

$$
B d y=C_{1} e^{C s} \int_{\partial Q}\left(\left|\partial_{t} \chi_{0}\right|^{2}+\left|\nabla \chi_{0}\right|^{2}+\left|\chi_{0}\right|^{2}\right)\left(\left|\nabla_{x, t} D_{t}^{\frac{1}{2}} u\right|^{2}+\left|D_{t}^{\frac{1}{2}} u\right|^{2}\right) \mathrm{d} \Sigma .
$$


However, constant $C_{1}$ now depends on $k$, which is the main difficulty in dealing with the equation (77) with the time-fractional derivative of irrational order by using the density of rational numbers in $\mathbb{R}$. The Carleman estimate for the equation (7) with the general order time-fractional derivatives remains open.

\subsection{Application to a lateral Cauchy problem for the sup-diffusion}

In this subsection, we employ the Carleman estimate in Theorem 3 to investigate the conditional stability for the lateral Cauchy problem. For this, we recall the partial differential equation:

$$
\partial_{t} u+q(x) \partial_{t}^{\frac{3}{4}} u-\Delta u+B \cdot \nabla u+c u=F \quad \text { in } Q
$$

with the zero initial condition:

$$
u(x, 0)=0, \quad x \in \Omega .
$$

We mainly follow the steps in [18] Theorem 5.1. Instead of a classical Carleman estimate for parabolic equation, we should apply our Carleman estimate established in Section 3.1

Proof of Theorem 4. By the choice of $\Omega_{0}$, we have $\overline{\Omega_{0}} \subset \Omega_{1}$ where $\Omega_{1}$ is defined in Section 1 Then we can find a sufficiently large $N>1$ such that

$$
\left\{x \in \Omega_{1}: d(x)>\frac{3}{N}\|d\|_{C\left(\overline{\Omega_{1}}\right)}\right\} \cap \bar{\Omega} \supset \Omega_{0} .
$$

Moreover we choose $\beta>0$ such that

$$
\beta \epsilon^{2} \leq\|d\|_{C\left(\overline{\Omega_{1}}\right)} \leq 2 \beta \epsilon^{2} .
$$

For any $t_{0} \in[\sqrt{2} \epsilon, T-\sqrt{2} \epsilon]$, we set $\mu_{k}:=\exp \left\{\frac{k}{N}\|d\|_{C\left(\overline{\Omega_{1}}\right)}-\frac{\beta \epsilon^{2}}{N}+c_{0}\right\}, E_{k}:=\left\{(x, t) \in Q_{1}: \varphi_{2}(x, t)>\mu_{k}\right\}$ and $D_{k}:=E_{k} \cap Q, k=1,2,3$. Recall that $c_{0}$ is the same constant as that in (10). Then we can easily verify the following two facts:

(i) $\Omega_{0} \times\left(t_{0}-\frac{\epsilon}{\sqrt{N}}, t_{0}+\frac{\epsilon}{\sqrt{N}}\right) \subset D_{3} \subset D_{2} \subset D_{1} \subset \bar{\Omega} \times\left(t_{0}-\sqrt{2} \epsilon, t_{0}+\sqrt{2} \epsilon\right)$,

(ii) $\partial D_{1} \subset \Sigma_{1} \cup \Sigma_{2}, \quad \Sigma_{1} \subset \Gamma \times(0, T), \Sigma_{2}=\left\{(x, t) \in Q: \varphi_{2}(x, t)=\mu_{1}\right\}$.

Next, we specify the cut-off function $\chi_{0}$ in Theorem 3 with $D_{0}=E_{2}$ and $D=E_{1}$. In detail, $\chi_{0} \in$ $C^{\infty}\left(\mathbb{R}^{n+1}\right)$ such that $0 \leq \chi_{0} \leq 1$ and

$$
\chi_{0}(x, t)= \begin{cases}1, & \varphi_{2}(x, t)>\mu_{2}, \\ 0, & \varphi_{2}(x, t) \leq \mu_{1} .\end{cases}
$$

Thus from Theorem 3 (denominator $k=4$ in this proof), it follows that

$$
\begin{aligned}
\int_{D_{1}} \chi_{0}^{2}\left(\sum_{j=-1}^{6} s^{2-j}\left|D_{t}^{\frac{j}{4}} u\right|^{2}+|\nabla u|^{2}+s^{-2} \sum_{i, j=1}^{n}\left|\partial_{i} \partial_{j} u\right|^{2}\right) e^{2 s \varphi_{2}} \mathrm{~d} x \mathrm{~d} t \\
\leq C \int_{D_{1}} \chi_{0}^{2}\left(\sum_{j=-1}^{2}\left|D_{t}^{\frac{j}{4}} F\right|^{2}\right) e^{2 s \varphi_{2}} \mathrm{~d} x \mathrm{~d} t+\text { Low }+ \text { Bdy }
\end{aligned}
$$

for all $s \geq s_{0}$ where Low and $B d y$ is the same notations as (11)-(12). In the above inequality, we have used the fact that $\varphi_{2}$ is upper and lower bounded by a constant which is independent of $s$. Since the second term Low on the RHS includes some derivates of $\chi_{0}$, it vanishes in $\overline{E_{2}}$ and outside of $E_{1}$, which implies that $\varphi_{2}(x, t) \leq \mu_{2}$ in $E_{1} \backslash \overline{E_{2}}\left(\supset D_{1} \backslash \overline{D_{2}}\right)$ and thus reads

$$
\text { Low }=C s \int_{Q}\left(\left|\partial_{t} \chi_{0}\right|^{2}+\left|\nabla \chi_{0}\right|^{2}+\sum_{i, j=1}^{n}\left|\partial_{i} \partial_{j} \chi_{0}\right|^{2}\right) \sum_{j=-1}^{2}\left(\left|\nabla\left(D_{t}^{\frac{j}{4}} u\right)\right|^{2}+\left|D_{t}^{\frac{j}{4}} u\right|^{2}\right) \mathrm{e}^{2 s \varphi_{2}} \mathrm{~d} x \mathrm{~d} t
$$




$$
\leq C \operatorname{se}^{2 s \mu_{2}} \int_{D_{1} \backslash \overline{D_{2}}} \sum_{j=-1}^{2}\left(\left|\nabla\left(D_{t}^{\frac{j}{4}} u\right)\right|^{2}+\left|D_{t}^{\frac{j}{4}} u\right|^{2}\right) \mathrm{d} x \mathrm{~d} t \leq C \operatorname{se}^{2 s \mu_{2}}\left\|D_{t}^{\frac{1}{2}} u\right\|_{H^{1,0}(Q)}^{2} .
$$

The last inequality above holds after the estimate (40). Furthermore, fact (ii) and the choice of $\chi_{0}$ yield that $\nabla \chi_{0}, \partial_{t} \chi_{0}$ and $\chi_{0}$ vanish on both $\Omega \times\{0, T\}$ and $(\partial \Omega \backslash \Gamma) \times(0, T)$ which indicate that

$$
\begin{aligned}
B d y & =C \mathrm{e}^{C s} \int_{\partial Q}\left(\left|\partial_{t} \chi_{0}\right|^{2}+\left|\nabla \chi_{0}\right|^{2}+\left|\chi_{0}\right|^{2}\right) \sum_{j=-1}^{2}\left(\left|\nabla_{x, t}\left(D_{t}^{\frac{j}{4}} u\right)\right|^{2}+\left|D_{t}^{\frac{j}{4}} u\right|^{2}\right) \mathrm{d} S \mathrm{~d} t \\
& \leq C \mathrm{e}^{C s} \int_{\Gamma \times(0, T)} \sum_{j=-1}^{2}\left(\left|\nabla_{x, t}\left(D_{t}^{\frac{j}{4}} u\right)\right|^{2}+\left|D_{t}^{\frac{j}{4}} u\right|^{2}\right) \mathrm{d} S \mathrm{~d} t \\
& \leq C \mathrm{e}^{C s}\left(\left\|\nabla_{x, t}\left(D_{t}^{\frac{1}{2}} u\right)\right\|_{L^{2}(\Gamma \times(0, T))}^{2}+\left\|D_{t}^{\frac{1}{2}} u\right\|_{L^{2}(\Gamma \times(0, T))}^{2}\right) .
\end{aligned}
$$

The last inequality again comes from (40). Thus, we obtain

$$
\begin{aligned}
R H S \text { of (45) } \leq & C \mathrm{e}^{C s} \sum_{j=-1}^{2} \int_{D_{1}}\left|D_{t}^{\frac{j}{4}} F\right|^{2} \mathrm{~d} x \mathrm{~d} t+C s \mathrm{e}^{2 s \mu_{2}}\left\|D_{t}^{\frac{1}{2}} u\right\|_{H^{1,0}(Q)}^{2} \\
& +C \mathrm{e}^{C s}\left(\left\|\nabla_{x, t}\left(D_{t}^{\frac{1}{2}} u\right)\right\|_{L^{2}(\Gamma \times(0, T))}^{2}+\left\|D_{t}^{\frac{1}{2}} u\right\|_{L^{2}(\Gamma \times(0, T))}^{2}\right) \\
\leq & C s \mathrm{e}^{2 s \mu_{2}} M^{2}+C \mathrm{e}^{C s} \mathcal{D}^{2}
\end{aligned}
$$

where $M$ and $\mathcal{D}$ are defined in Theorem 4 . On the other hand, the inclusion $\Omega_{0} \times\left(t_{0}-\frac{\epsilon}{\sqrt{N}}, t_{0}+\frac{\epsilon}{\sqrt{N}}\right) \subset$ $D_{3} \subset D_{2}$ and $\chi_{0}=1$ in $D_{2}$ imply that

$$
\begin{aligned}
\text { LHS of (45) } & =\int_{D_{2}}\left(\sum_{j=-1}^{6} s^{2-j}\left|D_{t}^{\frac{j}{4}} u\right|^{2}+|\nabla u|^{2}+s^{-2} \sum_{i, j=1}^{n}\left|\partial_{i} \partial_{j} u\right|^{2}\right) e^{2 s \varphi_{2}} \mathrm{~d} x \mathrm{~d} t \\
& \geq \int_{D_{3}}\left(\sum_{j=-1}^{6} s^{2-j}\left|D_{t}^{\frac{j}{4}} u\right|^{2}+|\nabla u|^{2}+s^{-2} \sum_{i, j=1}^{n}\left|\partial_{i} \partial_{j} u\right|^{2}\right) e^{2 s \varphi_{2}} \mathrm{~d} x \mathrm{~d} t \\
& \geq e^{2 s \mu_{3}} \int_{t_{0}-\frac{\epsilon}{\sqrt{N}}}^{t_{0}+\frac{\epsilon}{\sqrt{N}}} \int_{\Omega_{0}}\left(\sum_{j=-1}^{6} s^{2-j}\left|D_{t}^{\frac{j}{4}} u\right|^{2}+|\nabla u|^{2}+s^{-2} \sum_{i, j=1}^{n}\left|\partial_{i} \partial_{j} u\right|^{2}\right) \mathrm{d} x \mathrm{~d} t .
\end{aligned}
$$

Therefore (45) yields

$$
\|u\|_{H^{2,1}\left(\Omega_{0} \times\left(t_{0}-\frac{\epsilon}{\sqrt{N}}, t_{0}+\frac{\epsilon}{\sqrt{N}}\right)\right)}^{2} \leq C s^{3} e^{-2 s\left(\mu_{3}-\mu_{2}\right)} M^{2}+C s^{2} e^{C s} \mathcal{D}^{2}
$$

for all $s \geq s_{0}$. Since $\sup _{s>0} s^{3} e^{-s\left(\mu_{3}-\mu_{2}\right)}<\infty$ and $s^{2} \leq e^{C s}$ for $s$ large enough (e.g. $s \geq s_{1}$ ), we have

$$
\|u\|_{H^{2,1}\left(\Omega_{0} \times\left(t_{0}-\frac{\epsilon}{\sqrt{N}}, t_{0}+\frac{\epsilon}{\sqrt{N}}\right)\right)}^{2} \leq C e^{-C_{0} s} M^{2}+C e^{C s} \mathcal{D}^{2}
$$

for all $s \geq s_{2}:=\max \left\{s_{0}, s_{1}\right\}, C_{0}:=\mu_{3}-\mu_{2}>0$. After the change of the variable $s-s_{2} \rightarrow s$, we obtain

$$
\|u\|_{H^{2,1}\left(\Omega_{0} \times\left(t_{0}-\frac{\epsilon}{\sqrt{N}}, t_{0}+\frac{\epsilon}{\sqrt{N}}\right)\right)}^{2} \leq C e^{-C_{0} s} M^{2}+C e^{C s} \mathcal{D}^{2}
$$

for all $s \geq 0$. The generic constant $C$ depends on $s_{2}, \epsilon$ and the choice of $\Omega_{0}$, etc., but is still independent of $s$.

Let $m \in \mathbb{N}$ satisfy

$$
\sqrt{2} \epsilon+\frac{m \epsilon}{\sqrt{N}} \leq T-\sqrt{2} \epsilon<\sqrt{2} \epsilon+\frac{(m+1) \epsilon}{\sqrt{N}}<T .
$$

We here notice that the constant $C$ is also independent of $t_{0}$ provided that $t_{0} \in[\sqrt{2} \epsilon, T-\sqrt{2} \epsilon]$. Thus, by taking $t_{0}=\sqrt{2} \epsilon+\frac{k \epsilon}{\sqrt{N}}, k=0,1,2, \ldots, m$ in (46), summing up over $k$ and replacing $\sqrt{2} \epsilon$ by $\epsilon$, we obtain

$$
\|u\|_{H^{2,1}\left(\Omega_{0} \times(\epsilon, T-\epsilon)\right)}^{2} \leq C e^{-C_{0} s} M^{2}+C e^{C s} \mathcal{D}^{2}
$$


for all $s \geq 0$.

Finally, we show the estimate of Hölder type.

Firstly, let $\mathcal{D}=0$. Then letting $s \rightarrow \infty$ in (47), we see that $u=0$ in $\Omega_{0} \times(\epsilon, T-\epsilon)$. So that the conclusion holds true.

Secondly, let $\mathcal{D} \neq 0$. We divide it into two cases. In the case of $\mathcal{D} \geq M$, we see that (47) implies $\|u\|_{H^{2,1}\left(\Omega_{0} \times(\epsilon, T-\epsilon)\right)} \leq C e^{C s} \mathcal{D}$ for all $s \geq 0$. This has already proved the theorem. Now if $\mathcal{D} \leq M$. We choose $s>0$ minimizing the right-hand side of (47), that is,

$$
e^{-C_{0} s} M^{2}=e^{C s} \mathcal{D}^{2},
$$

which yields

$$
s=\frac{2}{C+C_{0}} \log \frac{M}{\mathcal{D}}
$$

in view of that $\mathcal{D} \neq 0$. Then (47) gives

$$
\|u\|_{H^{2,1}\left(\Omega_{0} \times(\epsilon, T-\epsilon)\right)}^{2} \leq 2 C M^{\frac{C}{C+C_{0}}} \mathcal{D}^{\frac{C_{0}}{C+C_{0}}} .
$$

The proof of Theorem 4 is completed.

\subsection{Application to an inverse source problem for the sup-diffusion}

In this subsection, we consider another application of Theorem 3. say, the stability for the inverse source problem (Problem 11). We point out here that the uniqueness result for this kind of inverse problem can be proved by a similar argument in 7 .

Before giving the proof of Theorem 5 we first recall the notation of the bounded domain $\Omega_{1}$ defined in (3), and function $d \in C^{2}\left(\overline{\Omega_{1}}\right)$ can be chosen so that (4) holds. Next for any $\epsilon>0$, we recall the level set of $\epsilon$ related to function $d$ as follows

$$
\Omega_{\epsilon}:=\{x \in \Omega: d(x)>\epsilon\} .
$$

Note that $\Omega_{\epsilon} \neq \emptyset$ for small $\epsilon$ and $\overline{\Omega_{\epsilon}} \cap \partial \Omega \subset \Gamma$. Now we are ready to give a proof of the stability result.

Proof of Theorem 5. Recall that our weight function is chosen as

$$
\varphi_{2}(x, t)=e^{\lambda \psi_{2}(x, t)}, \quad \psi_{2}(x, t)=d(x)-\beta\left(t-t_{0}\right)^{2}+c_{0} .
$$

Here, $c_{0}$ is a constant such that $\psi_{2}$ is always nonnegative and $s, \lambda$ are two large parameters while the parameter $\beta>0$ will be fixed later. Then on both sides of our governing equation (13), we integrate over the domain $\Omega_{3 \epsilon}$ at time $t=t_{0}$ and obtain

$$
\begin{gathered}
\int_{\Omega_{3 \epsilon}}\left|R\left(x, t_{0}\right) f(x)\right|^{2} e^{2 s \varphi_{2}\left(x, t_{0}\right)} \mathrm{d} x \leq \int_{\Omega_{3 \epsilon}}\left|\partial_{t} u\left(x, t_{0}\right)\right|^{2} \mathrm{e}^{2 s \varphi_{2}\left(x, t_{0}\right)} \mathrm{d} x+C \int_{\Omega_{3 \epsilon}}\left|D_{t}^{\frac{3}{4}} u\left(x, t_{0}\right)\right|^{2} \mathrm{e}^{2 s \varphi_{2}\left(x, t_{0}\right)} \mathrm{d} x \\
+\int_{\Omega_{3 \epsilon}}\left|-\Delta u\left(x, t_{0}\right)+B(x) \cdot \nabla u\left(x, t_{0}\right)+c(x) u\left(x, t_{0}\right)\right|^{2} \mathrm{e}^{2 s \varphi_{2}\left(x, t_{0}\right)} \mathrm{d} x .
\end{gathered}
$$

Easily, we see that the third term on the RHS is bounded by $C e^{C s}\left\|u\left(\cdot, t_{0}\right)\right\|_{H^{2}\left(\Omega_{3 \epsilon}\right)}^{2}$ and the LHS can be estimated with some $C_{0}>0$ :

$$
\int_{\Omega_{3 \epsilon}}\left|R\left(x, t_{0}\right) f(x)\right|^{2} e^{2 s \varphi_{2}\left(x, t_{0}\right)} d x \geq C_{0} \int_{\Omega_{3 \epsilon}}|f(x)|^{2} e^{2 s \varphi_{2}\left(x, t_{0}\right)} d x
$$

under the first assumption in (14). The key point is how to estimate the first and second terms on the RHS of (48).

By our notation $\Omega_{\epsilon}=\{x \in \Omega: d(x)>\epsilon\}$ for any $\epsilon>0$, we further set

$$
Q_{\epsilon}:=\left\{(x, t) \in Q: \psi_{2}(x, t)>\epsilon+c_{0}\right\}, \quad \epsilon>0 .
$$

Then we have the following relations: 
(i) $Q_{\epsilon} \subset \Omega_{\epsilon} \times(0, T)$,

(ii) $Q_{\epsilon} \supset \Omega_{\epsilon} \times\left\{t_{0}\right\}$.

In fact, if $(x, t) \in Q_{\epsilon}$, we have $d(x)-\beta\left(t-t_{0}\right)^{2}>\epsilon$, i.e. $d(x)>\beta\left(t-t_{0}\right)^{2}+\epsilon>\epsilon$. This means $x \in \Omega_{\epsilon}$. (i) is verified. On the other hand, if $x \in \Omega_{\epsilon}$ and $t=t_{0}$ then $\psi_{2}(x, t)=d(x)-\beta\left(t-t_{0}\right)^{2}+c_{0}=d(x)+c_{0}>\epsilon+c_{0}$. That is, $(x, t) \in Q_{\epsilon}$. (ii) is verified. Furthermore, we choose $\beta=\frac{\|d\|_{C\left(\overline{\Omega_{1}}\right)}}{\delta^{2}}$ where $\delta:=\min \left\{t_{0}, T-t_{0}\right\}$ so that

(iii) $\overline{Q_{\epsilon}} \cap(\Omega \times\{0, T\})=\emptyset$

is valid. Indeed, for $\forall(x, t) \in \Omega \times\{0, T\}, \psi(x, t)=d(x)-\beta\left(t-t_{0}\right)^{2}+c_{0} \leq\|d\|_{C\left(\overline{\Omega_{1}}\right)}-\beta \delta^{2}+c_{0}=c_{0}$. This leads to $(x, t) \notin \overline{Q_{\epsilon}}$.

Relations (i) - (iii) guarantee that $Q_{\epsilon}$ is a sub-domain of $Q$ and $\partial Q_{\epsilon} \cap \partial Q \subset \Gamma \times(0, T)$. Moreover, we assert that

(iv) $\Omega_{3 \epsilon} \times\left(t_{0}-\delta_{\epsilon}, t_{0}\right) \subset Q_{2 \epsilon}, \quad \delta_{\epsilon}:=\sqrt{\frac{\epsilon}{\beta}}=\sqrt{\frac{\epsilon}{\|d\|}} \delta$.

Actually, for any $(x, t) \in \Omega_{3 \epsilon} \times\left(t_{0}-\delta_{\epsilon}, t_{0}\right)$, we have

$$
\psi(x, t)=d(x)-\beta\left(t-t_{0}\right)^{2}+c_{0}>3 \epsilon-\beta \delta_{\epsilon}^{2}+c_{0}=2 \epsilon+c_{0} .
$$

That is, $(x, t) \in Q_{2 \epsilon}$.

Now we construct a function $\eta \in C^{2}[0, T]$ such that $0 \leq \eta \leq 1$ and

$$
\eta=\left\{\begin{array}{l}
1 \quad \text { in }\left[t_{0}-\frac{1}{2} \delta_{\epsilon}, t_{0}+\frac{1}{2} \delta_{\epsilon}\right], \\
0 \quad \text { in }\left[0, t_{0}-\delta_{\epsilon}\right] \cup\left[t_{0}+\delta_{\epsilon}, T\right]
\end{array}\right.
$$

for any small $\epsilon<\|d\|_{C\left(\overline{\Omega_{1}}\right)}$. Then by the use of the condition (15), and noting that $\eta\left(t_{0}-\delta_{\epsilon}\right)=0, \eta\left(t_{0}\right)=1$, we have

$$
\begin{aligned}
\int_{\Omega_{3 \epsilon}}\left|\partial_{t} u\left(x, t_{0}\right)\right|^{2} e^{2 s \varphi_{2}\left(x, t_{0}\right)} \mathrm{d} x & =\int_{\Omega_{3 \epsilon}}\left|\eta\left(t_{0}\right) \partial_{t} u\left(x, t_{0}\right)\right|^{2} \mathrm{e}^{2 s \varphi_{2}\left(x, t_{0}\right)} \mathrm{d} x \\
& =\int_{t_{0}-\delta_{\epsilon}}^{t_{0}} \frac{\mathrm{d}}{\mathrm{d} t} \int_{\Omega_{3 \epsilon}}\left|\eta \partial_{t} u\right|^{2} \mathrm{e}^{2 s \varphi_{2}} \mathrm{~d} x \mathrm{~d} t \\
& =\int_{t_{0}-\delta_{\epsilon}}^{t_{0}} \int_{\Omega_{3 \epsilon}} 2 \eta \partial_{t} u\left(\eta \partial_{t}^{2} u+\partial_{t} \eta \partial_{t} u\right) \mathrm{e}^{2 s \varphi_{2}} \mathrm{~d} x \mathrm{~d} t \\
& -\int_{t_{0}-\delta_{\epsilon}}^{t_{0}} \int_{\Omega_{3 \epsilon}} 4\left(t-t_{0}\right) \beta s \lambda \varphi_{2}\left|\eta \partial_{t} u\right|^{2} \mathrm{e}^{2 s \varphi_{2}} \mathrm{~d} x \mathrm{~d} t \\
\leq & C \int_{Q_{2 \epsilon}}\left(s^{-2}\left|\partial_{t}^{2} u\right|^{2}+s^{2}\left|\partial_{t} u\right|^{2}\right) \mathrm{e}^{2 s \varphi_{2}} \mathrm{~d} x \mathrm{~d} t
\end{aligned}
$$

and

$$
\begin{aligned}
\int_{\Omega_{3 \epsilon}}\left|D_{t}^{\frac{3}{4}} u\left(x, t_{0}\right)\right|^{2} e^{2 s \varphi_{2}\left(x, t_{0}\right)} \mathrm{d} x & =\int_{\Omega_{3 \epsilon}}\left|\eta\left(t_{0}\right) D_{t}^{\frac{3}{4}} u\left(x, t_{0}\right)\right|^{2} e^{2 s \varphi_{2}\left(x, t_{0}\right)} \mathrm{d} x \\
= & \int_{t_{0}-\delta_{\epsilon}}^{t_{0}} \frac{\mathrm{d}}{\mathrm{d} t} \int_{\Omega_{3 \epsilon}}\left|\eta D_{t}^{\frac{3}{4}} u\right|^{2} \mathrm{e}^{2 s \varphi_{2}} \mathrm{~d} x \mathrm{~d} t \\
= & \int_{t_{0}-\delta_{\epsilon}}^{t_{0}} \int_{\Omega_{3 \epsilon}} 2 \eta D_{t}^{\frac{3}{4}} u\left(\eta \partial_{t}\left(D_{t}^{\frac{3}{4}} u\right)+\partial_{t} \eta D_{t}^{\frac{3}{4}} u\right) \mathrm{e}^{2 s \varphi_{2}} \mathrm{~d} x \mathrm{~d} t \\
& -\int_{t_{0}-\delta_{\epsilon}}^{t_{0}} \int_{\Omega_{3 \epsilon}} 4 \beta s \lambda \varphi_{2}\left(t-t_{0}\right)\left|\eta D_{t}^{\frac{3}{4}} u\right|^{2} \mathrm{e}^{2 s \varphi_{2}} \mathrm{~d} x \mathrm{~d} t \\
\leq & \frac{C}{s} \int_{Q_{2 \epsilon}}\left(s^{-1}\left|D_{t}^{\frac{7}{4}} u\right|^{2}+s^{3}\left|D_{t}^{\frac{3}{4}} u\right|^{2}\right) \mathrm{e}^{2 s \varphi_{2}} \mathrm{~d} x \mathrm{~d} t .
\end{aligned}
$$

Now we intend to use the Carleman estimate established in Section 3.1 to evaluate the RHS of (50) and (51). For this, we take time derivative on both sides of (13) and then $u_{1}:=\partial_{t} u$ reads the following equation

$$
\partial_{t} u_{1}+q(x) \partial_{t}^{\frac{3}{4}} u_{1}-\Delta u_{1}+B \cdot \nabla u_{1}+c u_{1}=\left(\partial_{t} R\right) f, \quad \text { in } Q .
$$


Noting the compatible condition $R(\cdot, 0)=0$, we apply Theorem 3 to (52), and then we have

$$
\begin{aligned}
& \int_{Q} \chi_{0}^{2}\left(s^{-2}\left|\partial_{t} u_{1}\right|^{2}+s^{-1}\right.\left.\left|D_{t}^{\frac{3}{4}} u_{1}\right|^{2}+s^{2}\left|u_{1}\right|^{2}+s^{3}\left|D_{t}^{-\frac{1}{4}} u_{1}\right|^{2}\right) e^{2 s \varphi_{2}} \mathrm{~d} x \mathrm{~d} t \\
& \leq C \int_{Q} \chi_{0}^{2}\left(\sum_{j=3}^{6}\left|D_{t}^{\frac{j}{4}} R\right|^{2}\right)|f|^{2} e^{2 s \varphi_{2}} \mathrm{~d} x \mathrm{~d} t+\text { Low } 1+B d y 1
\end{aligned}
$$

for all $s \geq s_{1} \geq 1$, where the terms Low1 and Bdy1 are defined as

$$
\begin{aligned}
& \text { Low } 1=C s \int_{Q}\left(\left|\partial_{t} \chi_{0}\right|^{2}+\left|\nabla \chi_{0}\right|^{2}+\sum_{i, j=1}^{n}\left|\partial_{i} \partial_{j} \chi_{0}\right|^{2}\right)\left(\sum_{j=-1}^{2}\left(\left|\nabla\left(D_{t}^{\frac{j}{4}} u_{1}\right)\right|^{2}+\left|D_{t}^{\frac{j}{4}} u_{1}\right|^{2}\right)\right) e^{2 s \varphi_{2}} \mathrm{~d} x \mathrm{~d} t, \\
& B d y 1=C e^{C s} \int_{\partial Q}\left(\left|\partial_{t} \chi_{0}\right|^{2}+\left|\nabla \chi_{0}\right|^{2}+\left|\chi_{0}\right|^{2}\right) \sum_{j=-1}^{2}\left(\left|\nabla_{x, t}\left(D_{t}^{\frac{j}{4}} u_{1}\right)\right|^{2}+\left|D_{t}^{\frac{j}{4}} u_{1}\right|^{2}\right) \mathrm{d} S \mathrm{~d} t .
\end{aligned}
$$

We choose the cut-off function $\chi_{0} \in C^{\infty}\left(\mathbb{R}^{n+1}\right)$ such that $0 \leq \chi_{0} \leq 1$ and

$$
\chi_{0}= \begin{cases}1, & \psi_{2}(x, t)>2 \epsilon+c_{0}, \\ 0, & \psi_{2}(x, t) \leq \epsilon+c_{0} .\end{cases}
$$

Therefore $\chi_{0}=1$ in $Q_{2 \epsilon}$ while its derivatives vanish in $Q_{2 \epsilon}$ which enable us to rewrite the above Carleman inequality as follows:

$$
\begin{aligned}
\int_{Q_{2 \epsilon}}\left(s^{-2}\left|\partial_{t}^{2} u\right|^{2}+s^{-1}\left|D_{t}^{\frac{7}{4}} u\right|^{2}+s^{2}\left|\partial_{t} u\right|^{2}+s^{3}\left|D_{t}^{\frac{3}{4}} u\right|^{2}\right) e^{2 s \varphi_{2}} \mathrm{~d} x \mathrm{~d} t \\
\leq C \int_{Q_{\epsilon}}\left(\sum_{j=3}^{6}\left|D_{t}^{\frac{j}{4}} R\right|^{2}\right)|f|^{2} e^{2 s \varphi_{2}} \mathrm{~d} x \mathrm{~d} t+\text { Low } 2+\text { Bdy2 }
\end{aligned}
$$

for all $s \geq s_{1} \geq 1$, where

$$
\begin{aligned}
& \text { Low } 2=C s e^{2 s e^{\lambda\left(2 \epsilon+c_{0}\right)}} \int_{Q_{\epsilon} \backslash Q_{2 \epsilon}}\left(\left|\nabla\left(D_{t}^{\frac{3}{2}} u\right)\right|^{2}+\left|D_{t}^{\frac{3}{2}} u\right|^{2}\right) \mathrm{d} x \mathrm{~d} t, \\
& B d y 2=C e^{C s} \int_{\Gamma \times(0, T)}\left(\left|\nabla_{x, t}\left(D_{t}^{\frac{3}{2}} u\right)\right|^{2}+\left|D_{t}^{\frac{3}{2}} u\right|^{2}\right) \mathrm{d} S \mathrm{~d} t .
\end{aligned}
$$

The above inequality is derived from the choice of $\chi_{0}$ and the estimate (40) as well as the relations (i)-(iii) which indicate that both the derivatives of $\chi_{0}$ and $\chi_{0}$ itself vanish on $\partial Q$ except for the lateral boundary $\Gamma \times(0, T)$. Now substituting (53) into (50) and (51) implies that

$$
\begin{aligned}
\int_{\Omega_{3 \epsilon}}\left|\partial_{t} u\left(x, t_{0}\right)\right|^{2} e^{2 s \varphi_{2}\left(x, t_{0}\right)} \mathrm{d} x+\int_{\Omega_{3 \epsilon}}\left|D_{t}^{\frac{3}{4}} u\left(x, t_{0}\right)\right|^{2} e^{2 s \varphi_{2}\left(x, t_{0}\right)} \mathrm{d} x \\
\leq C \int_{Q_{\epsilon}}\left(\sum_{j=3}^{6}\left|D_{t}^{\frac{j}{4}} R\right|^{2}\right)|f|^{2} e^{2 s \varphi_{2}} \mathrm{~d} x \mathrm{~d} t+\text { Low } 2+\text { Bdy2. }
\end{aligned}
$$

Combined with (48) and (49), we obtain

$$
\int_{\Omega_{3 \epsilon}}|f(x)|^{2} \mathrm{e}^{2 s \varphi_{2}\left(x, t_{0}\right)} \mathrm{d} x \leq C \mathrm{e}^{C s}\left\|u\left(\cdot, t_{0}\right)\right\|_{H^{2}\left(\Omega_{3 \epsilon}\right)}^{2}+C \int_{Q_{\epsilon}}|f|^{2} \mathrm{e}^{2 s \varphi_{2}} \mathrm{~d} x \mathrm{~d} t+\text { Low } 2+B d y 2 .
$$

Moreover, we divide the second term on the RHS into two parts:

$$
C \int_{Q_{\epsilon}}|f|^{2} \mathrm{e}^{2 s \varphi_{2}} \mathrm{~d} x \mathrm{~d} t=C \int_{Q_{3 \epsilon}}|f|^{2} \mathrm{e}^{2 s \varphi_{2}} \mathrm{~d} x \mathrm{~d} t+C \int_{Q_{\epsilon} \backslash Q_{3 \epsilon}}|f|^{2} \mathrm{e}^{2 s \varphi_{2}} \mathrm{~d} x \mathrm{~d} t
$$




$$
\leq C \int_{Q_{3 \epsilon}}|f|^{2} \mathrm{e}^{2 s \varphi_{2}} \mathrm{~d} x \mathrm{~d} t+C \mathrm{e}^{2 s \mathrm{e}^{\lambda\left(3 \epsilon+c_{0}\right)}} \int_{Q_{\epsilon} \backslash Q_{3 \epsilon}}|f|^{2} \mathrm{~d} x \mathrm{~d} t
$$

which leads to

$$
\begin{aligned}
\int_{\Omega_{3 \epsilon}}|f(x)|^{2} \mathrm{e}^{2 s \varphi_{2}\left(x, t_{0}\right)} \mathrm{d} x & \leq C \int_{Q_{3 \epsilon}}|f|^{2} \mathrm{e}^{2 s \varphi_{2}} \mathrm{~d} x \mathrm{~d} t+C \mathrm{e}^{2 s \mathrm{e}^{\lambda\left(3 \epsilon+c_{0}\right)}} \int_{Q_{\epsilon} \backslash Q_{3 \epsilon}}|f|^{2} \mathrm{~d} x \mathrm{~d} t \\
& +C \mathrm{e}^{2 s \mathrm{e}^{\lambda\left(2 \epsilon+c_{0}\right)}} \int_{Q_{\epsilon}}\left(\left|\nabla\left(D_{t}^{\frac{3}{2}} u\right)\right|^{2}+\left|D_{t}^{\frac{3}{2}} u\right|^{2}\right) \mathrm{d} x \mathrm{~d} t+C \mathrm{e}^{C s} \mathcal{D}^{2} \\
& \leq C \int_{Q_{3 \epsilon}}|f|^{2} \mathrm{e}^{2 s \varphi_{2}} \mathrm{~d} x \mathrm{~d} t+C \mathrm{e}^{2 s \mathrm{e}^{\lambda\left(3 \epsilon+c_{0}\right)}} M^{2}+C \mathrm{e}^{C s} \mathcal{D}^{2}
\end{aligned}
$$

for all $s \geq s_{1} \geq 1$. Here $M$ and $\mathcal{D}$ denote a priori bound and measurements defined in Theorem 5 , Since $\varphi_{2}(x, t)$ attains its maximum at $t=t_{0}$, we can absorb the first term on the RHS into the LHS by taking $s$ large enough (e.g. $s \geq s_{2}$ ). That is

$$
\int_{\Omega_{3 \epsilon}}|f(x)|^{2} \mathrm{e}^{2 s \varphi_{2}\left(x, t_{0}\right)} \mathrm{d} x \leq C s e^{2 s e^{\lambda\left(3 \epsilon+c_{0}\right)}} M^{2}+C e^{C s} \mathcal{D}^{2}
$$

for all $s \geq s_{3}=\max \left\{s_{1}, s_{2}\right\}$. On the hand hand,

$$
\int_{\Omega_{3 \epsilon}}|f(x)|^{2} e^{2 s \varphi_{2}\left(x, t_{0}\right)} \mathrm{d} x \geq \int_{\Omega_{4 \epsilon}}|f(x)|^{2} e^{2 s \varphi_{2}\left(x, t_{0}\right)} \mathrm{d} x \geq e^{2 s e^{\lambda\left(4 \epsilon+c_{0}\right)}}\|f\|_{L^{2}\left(\Omega_{4 \epsilon}\right)}^{2} .
$$

Therefore, we obtain

$$
\|f\|_{L^{2}\left(\Omega_{4 \epsilon}\right)}^{2} \leq C s e^{-2 \epsilon_{0} s} M^{2}+C e^{C s} \mathcal{D}^{2}
$$

for all $s \geq s_{3}$. Here $\epsilon_{0}:=e^{\lambda\left(4 \epsilon+c_{0}\right)}-e^{\lambda\left(3 \epsilon+c_{0}\right)}=e^{\lambda\left(3 \epsilon+c_{0}\right)}\left(e^{\lambda \epsilon}-1\right)>0$. Since $\sup _{s>0} s e^{-\epsilon_{0} s}<\infty$, the above inequality gives

$$
\|f\|_{L^{2}\left(\Omega_{4 \epsilon}\right)}^{2} \leq C e^{-\epsilon_{0} s} M^{2}+C e^{C s} \mathcal{D}^{2}
$$

for all $s \geq \hat{s}$ with $\hat{s} \geq s_{3}$ satisfying $\hat{s} e^{-\epsilon_{0} \hat{s}} \leq C$. By substituting $s$ by $s+\hat{s}$, inequality (54) holds for all $s \geq 0$ with a larger generic constant $C e^{\hat{s}}$ which is again denoted by $C$.

Finally, we repeat the argument in the proof of Theorem 4 to show the estimate of Hölder type:

$$
\|f\|_{L^{2}\left(\Omega_{4 \epsilon}\right)} \leq C\left(M^{1-\theta} \mathcal{D}^{\theta}+\mathcal{D}\right) .
$$

The proof of Theorem 5 is completed.

\section{Conclusions and remarks}

In this paper, we considered the Carleman estimates for the time-fractional advection-diffusion equation (2) and the applications.

First, in the case of sub-diffusion, that is, the largest fractional order is strictly less than half, the Carleman estimate for the equation (2) was established by regarding the fractional order terms as perturbation of the first order time-derivative and the use of the Carleman estimate for the parabolic equations. As an application, the conditional stability for a lateral Cauchy problem was obtained, say, the solution of the equation (2) is continuously dependent of not only the partial Cauchy data and the source term but also the initial value. Due to our choice of the weight function $\psi_{1}(x, t)=d(x)-\beta t^{2-2 \alpha_{1}}$, we do not know whether the estimate is valid without the initial value, and this remains open. On the other hand, the choice of the new weight function $\psi_{1}$ is not suitable for the study of the inverse problems. As is well known, for dealing with the inverse problems, the Carleman type estimate derived by $d(x)-\beta\left(t-t_{0}\right)^{2}+c_{0}\left(t_{0} \in(0, T)\right)$ should be better according to the series of theories in 18. The inverse problems for the equation (2) in the case of $\alpha_{1}<\frac{1}{2}$ remain open. The above arguments cannot deal with the case of sup-diffusion where the largest order is greater than half neither. However, in the case of the largest order is rational number and less than $\frac{3}{4}$, we found the Carleman estimate with a cut-off function for the equation (7) by using the regular weight 
function $d(x)-\beta\left(t-t_{0}\right)^{2}+c_{0}\left(t_{0} \in(0, T)\right)$ can be constructed. Then by an argument similar to that in [18, the conditional stability for the inverse source problem (Problem 1) was proved as well as the conditional stability for the lateral Cauchy problem. The fractional order $\alpha=\frac{3}{4}$ is the largest one which one can deal with based on our arguments of Carleman estimate. Moreover, constant $\hat{s}$ and the generic constant $C$ and in Theorem 3 depend on the denominator of fractional order $\alpha$ which prevents us from extending the order to real number by the density of $\mathbb{Q}$ in $\mathbb{R}$. The case of the general order remains open.

Finally, it should be mentioned that the stability inequalities in Theorem 4 immediately gives the unique continuation result of (7), i.e. the solution of the equation (7) must vanish in the whole domain $Q$ if its initial value is identically 0 in $\Omega$ and the partial boundary data $\left.u\right|_{\Gamma \times(0, T)}$ and $\left.\partial_{\nu} u\right|_{\Gamma \times(0, T)}$ are zero. This principle is called a weak type unique continuation for the equation (2) since the homogeneous initial value is not essential for the unique continuation (UC) (e.g., UC for the elliptic equations or UC for the parabolic equations). However, we cannot repeat this argument to derive the weak unique continuation of (2) because the constant $\varepsilon>0$ depends on the choice of $\Omega_{0}$ in the estimate in Theorem 2. We refer to [7] and [15] for other kind of weak type unique continuation where the initial value does not vanish but the homogeneous Dirichlet or Neumann boundary condition is required.

\section{Acknowledgement}

The first author thanks the Leading Graduate Course for Frontiers of Mathematical Sciences and Physics (FMSP, The University of Tokyo). The second author thanks Grant-in-Aid for Research Activity Start-up 16H06712, JSPS. This work was supported by A3 Foresight Program "Modeling and Computation of Applied Inverse Problems", Japan Society of the Promotion of Science (JSPS). The second and third authors are supported by Grant-in-Aid for Scientific Research (S) 15H05740, JSPS.

\section{References}

[1] Adams R A. Sobolev Spaces. Academic Press, New York, 1975.

[2] Benson D A, Wheatcraft S W, Meerschaert M M. Application of a fractional advection-dispersion equation. Water Resources Research, 2000, 36(6): 1403-1412.

[3] Cheng J, Lin C L, Nakamura G. Unique continuation property for the anomalous diffusion and its application. Journal of Differential Equations, 2013, 254(9): 3715-3728.

[4] Cheng J, Nakagawa J, Yamamoto M, et al. Uniqueness in an inverse problem for a one-dimensional fractional diffusion equation. Inverse problems, 2009, 25(11): 115002.

[5] Haggerty R, Wondzell S M, Johnson M A. Powerlaw residence time distribution in the hyporheic zone of a 2ndorder mountain stream. Geophysical Research Letters, 2002, 29(13).

[6] Y. Hatno and N. Hatano. Hatano Y, Hatano N. Dispersive transport of ions in column experiments: An explanation of longtailed profiles. Water Resources Research, 1998, 34(5): 1027-1033.

[7] Jiang D, Li Z, Liu Y, et al. Weak unique continuation property and a related inverse source problem for time-fractional diffusion-advection equations. Inverse Problems, 2017.

[8] Kawamoto A. Hölder stability estimate in an inverse source problem for a first and half order time fractional diffusion equation[J]. arXiv preprint arXiv:1611.05200, 2016.

[9] Kilbas A A A, Srivastava H M, Trujillo J J. Theory And Applications of Fractional Differential Equations. Elsevier Science Limited, 2006.

[10] Li Z, Imanuvilov O Y, Yamamoto M. Uniqueness in inverse boundary value problems for fractional diffusion equations. Inverse Problems, 2015, 32(1): 015004.

[11] Li Z, Yamamoto M. Uniqueness for inverse problems of determining orders of multi-term time-fractional derivatives of diffusion equation[J]. Applicable Analysis, 2015, 94(3): 570-579. 
[12] Levy M, Berkowitz B. Measurement and analysis of non-Fickian dispersion in heterogeneous porous media. Journal of contaminant hydrology, 2003, 64(3): 203-226.

[13] Mainardi F, Mura A, Pagnini G, et al. Time-fractional diffusion of distributed order. Journal of Vibration and Control, 2008, 14(9-10): 1267-1290.

[14] Podlubny I. Fractional differential equations: an introduction to fractional derivatives, fractional differential equations, to methods of their solution and some of their applications. Academic press, 1998.

[15] Sakamoto K, Yamamoto M. Initial value/boundary value problems for fractional diffusion-wave equations and applications to some inverse problems[J]. Journal of Mathematical Analysis and Applications, 2011, 382(1): 426-447.

[16] Schumer R, Benson D A, Meerschaert M M, et al. Fractal mobile/immobile solute transport. Water Resources Research, 2003, 39(10).

[17] Xu X, Cheng J, Yamamoto M. Carleman estimate for a fractional diffusion equation with half order and application[J]. Applicable Analysis, 2011, 90(9): 1355-1371.

[18] Yamamoto M. Carleman estimates for parabolic equations and applications. Inverse problems, 2009, 25(12): 123013

[19] Zhang Z. An undetermined coefficient problem for a fractional diffusion equation. Inverse Problems, 2015, 32(1): 015011. 